\title{
ON THE MEASURE OF CARTESIAN PRODUCT SETS
}

\author{
BY \\ GERALD FREILICH
}

1. Introduction. There are various measure functions defined on the set of all subsets of Euclidean $n$-space, $E^{n}$, which generalize the elementary concepts of length or area, or in general, $k$-dimensional volume. These measures are called respectively 1 -dimensional, 2-dimensional, and $k$-dimensional measures over $E^{n}$. The following question then naturally arises:

For $A$ and $B$ orthogonal subspaces of $E^{n}$ with dimensions $\alpha$ and $\beta=n-\alpha$, and for $0 \leqq m \leqq \alpha$ and $0 \leqq k \leqq \beta$, is the $(m+k)$-dimensional measure of the cartesian product of the finitely measurable sets $S \subset A$ and $T \subset B$ equal to the m-dimensional measure of $S$ times the $k$-dimensional measure of $T$ ?

In the case $n=3, \alpha=2, \beta=m=k=1$, and $T$ an interval, J. F. Randolph obtained a partial answer for Carathéodory and Gross measures (see $[R])\left({ }^{1}\right)$, A. P. Morse and Randolph obtained the affirmative answer for Gillespie measure (see $[M R]$ ), while A. S. Besicovitch and P. A. P. Moran presented a rough outline of a counter example for Hausdorff measure (see [BM]). By using new methods the current paper obtains results for integral-geometric (Favard) measure, Hausdorff measure, and sphere measure.

The cylinder case $(\beta=1)$ for Favard measure is answered in the affirmative (Theorem 4.5) and further generalized (Theorem 5.5) to $k=\beta$, thus completely answering the question if $n \leqq 3$. Partial results for the remaining cases are contained in Theorems 5.7 and 5.8.

In 6.10 an example is constructed, using and extending the ideas of Besicovitch and Moran, which answers the question in the negative for both Hausdorff measure (Theorem 6.18) and for sphere measure (Theorem 6.19). Moreover we obtain in this way a set for which the Hausdorff measure differs from the Carathéodory measure.

Finally we obtain for $X \subset E^{n}, k \leqq m \leqq n$, a new formula which expresses $\mathcal{F}_{n}^{k}(X)$, in terms of the multiplicity integrals, with respect to $\mathcal{F}_{m}^{k}$, of the perpendicular projections of $X$ into $m$ planes, as an integral over the set of all these $m$ planes $\left(\mathcal{F}_{n}^{k}\right.$ is Favard $k$ measure over $\left.E^{n}\right)$.

2. Preliminaries.

2.1 Definition. We agree that $\infty \cdot 0=0 \cdot \infty=0$.

For $S$ a set, $\gamma(S)$ is the number (possibly $\infty$ ) of elements of $S$. For $F$ a family of sets, $\sigma(F)=\bigcup_{x \in F} x$.

For $x$ a point,

Presented to the Society, April 30, 1949; received by the editors June 29, 1949.

(1) Symbols in brackets refer to the bibliography at the end of the paper. 


$$
\{x\}=\underset{y}{E}[y=x] \text {. }
$$

For $f$ a function, $f^{-1}$ is its inverse, and for $X$ a set, $(f \mid X)$ is the function with domain ( $X \cap$ domain $f$ ) defined by

$$
(f \mid X)(x)=f(x) \text { for } \quad x \in(X \cap \operatorname{domain} f) ;
$$

furthermore

$$
\begin{aligned}
N[f, X, x] & =\gamma(X \cap \underset{y}{E}[f(y)=x]), \\
f^{*}(X) & =\underset{y}{E}[y=f(x) \text { for some } x \in X],
\end{aligned}
$$

and for $g$ also a function, $(f: g)$ is the superposition function defined by $(f: g)(x)=f(g(x))$ for all $x$.

2.2 Definition. $E^{n}$ is Euclidean $n$-dimensional vector space with the usual inner product and metric. We denote the origin of $E^{n}$ by $\theta^{n}$. If $A \subset E^{n}$ and $B \subset E^{n}$, then

$$
A+B=E^{n} \cap \underset{z}{E}[z=x+y \text { for some } x \in A, y \in B] .
$$

Lebesgue $n$-dimensional measure over $E^{n}$ is denoted by $\mathcal{L}_{n}$.

2.3 Definition. If $m$ and $n$ are positive integers, and $L$ is a function on $E^{m}$ to $E^{n}$, then $L$ is linear if and only if

$$
\begin{aligned}
L(x+y) & =L(x)+L(y) & & \text { for } x \in E^{m}, y \in E^{m} \\
L(\lambda \cdot x) & =\lambda \cdot L(x) & & \text { for } x \in E^{m}, \lambda \text { a real number. }
\end{aligned}
$$

If $L$ is a linear function on $E^{m}$ to $E^{n}, 1 \leqq i \leqq m$, and $I^{i}$ is the $i$ th unit vector of $E^{m}$, then we shall write

$$
L\left(I^{i}\right)=L^{i}=\left(L_{1}^{i}, L_{2}^{i}, \cdots, L_{n}^{i}\right) .
$$

By the matrix of $L$ we mean the matrix with $n$ rows and $m$ columns whose entry in the $i$ th row and $j$ th column $(1 \leqq i \leqq n, 1 \leqq j \leqq m)$ is $L_{i}^{j}$. We make no distinction between $L$ and its matrix. If $m \leqq n$, we define

$\Delta(L)$ is the square root of the sum of the squares of the determinants of all $m$ by $m$ minors of $L$.

If $f$ is a function on $E^{m}$ to $E^{n}, 1 \leqq m \leqq n$, and $x \in E^{m}$, then we shall say that $L$ is the differential of $f$ at $x$ if and only if $L$ is a linear function on $E^{m}$ to $E^{n}$ such that

$$
\lim _{z \rightarrow x} \frac{|f(z)-f(x)-L(z-x)|}{|z-x|}=0 .
$$

If $L$ is the (unique) differential of $f$ at $x$, then we let

$$
J f(x)=\Delta(L)
$$


2.4 Definition. If $1 \leqq m \leqq n$, then $p_{n}^{m}$ is the function on $E^{n}$ to $E^{m}$ such that

$$
p_{n}^{m}(x)=\left(x_{1}, x_{2}, \cdots, x_{m}\right) \quad \text { for } x \in E^{n},
$$

and $\eta_{n}^{m}$ is the function on $E^{m}$ to $E^{n}$ such that

$$
\eta_{n}^{m}(y)=\left(y_{1}, y_{2}, \cdots, y_{m}, 0,0, \cdots, 0\right) \quad \text { for } y \in E^{m} .
$$

2.5 Definition. Let $G_{n}$ be the set of all linear functions $R$ on $E^{n}$ to $E^{n}$ such that $|R(x)|=|x|$ for $x \in E^{n}$. The function $\rho$ defined by the formula

$$
\rho(R, S)=\sup _{|x|=1}|R(x)-S(x)| \quad \text { for } R \dot{\epsilon} \in G_{n}, S \in G_{n},
$$

metrizes $G_{n}$ in such a way that $G_{n}$ is a compact topological group with respect to the operation, :, of superposition. $G_{n}$ is called the orthogonal group of $E^{n}$. We shall denote the identity of $G_{n}$ by ${ }^{n} I$.

We let $\phi_{n}$ denote the unique Haar measure over $G_{n}$ for which $\phi_{n}\left(G_{n}\right)=1$, nonempty open sets are $\phi_{n}$ measurable and have positive $\phi_{n}$ measure, and the $\phi_{n}$ measure of a subset of $G_{n}$ is the infimum of the $\phi_{n}$ measures of open sets containing it.

We shall use the following properties of $\phi_{n}$ (see $\left.[\mathrm{W}, 8]\right)$ :

If $f$ is a $\phi_{n}$ measurable function on $G_{n}$, then

$$
\int_{G_{n}} f\left(R^{-1}\right) d \phi_{n} R=\int_{G_{n}} f(R) d \phi_{n} R,
$$

and for $S \in G_{n}$,

$$
\int_{G_{n}} f(S: R) d \phi_{n} R=\int_{G_{n}} f(R: S) d \phi_{n} R=\int_{G_{n}} f(R) d \phi_{n} R .
$$

2.6 Definition. If $1 \leqq k \leqq n$, then

$$
\beta(n, k)=\int_{G_{n}} \Delta\left(\left.R\right|_{k} ^{k}\right) d \phi_{n} R,
$$

where $\left(\left.R\right|_{k} ^{k}\right)$ is defined for $R \in G_{n}$, as the linear function on $E^{k}$ to $E^{k}$ such that $\left(\left.R\right|_{k} ^{k}\right)_{j}^{i}=R_{j}^{i}$ for $i, j=1,2, \cdots, k$.

2.7 Definition. Let

$$
Z_{n}^{*}=E^{n} \cap \underset{z}{E}\left[x_{i}=0 \text { for } i=1,2, \cdots, n-s\right],
$$

and define the function $\lambda_{n}^{s}$ on the cartesian product $\left(G_{n} \times E^{n-s}\right)$ to the set $\Lambda_{n}^{s}$ of all $s$-dimensional flat subspaces of $E^{n}$ by the formula

$$
\lambda_{n}^{s}(R, w)=R^{*}\left(Z_{n}^{8}+\left\{\eta_{n}^{n-s}(w)\right\}\right) \text { for } R \in G_{n}, w \in E^{n-s} .
$$


The motivation for this definition rests on the following fact:

Let $\Psi_{n}^{s}(F)$ be defined for each suitably restricted real-valued function $F$ on $\Lambda_{n}^{s}$ by the formula

$$
\Psi_{n}^{8}(F)=\int_{G_{n}} \int_{E^{n-s}} F\left(\lambda_{n}^{8}(R, w)\right) d \mathcal{L}_{n-8} w d \phi_{n} R .
$$

Then $\Psi_{n}^{s}$ is an invariant integral. (See $[F 3,4]$.)

2.8 Definition. We say that $g$ is a gauge over $S$ if and only if $S$ is a metric space,

$$
\operatorname{dmn} g \subset \underset{x}{E}[x \subset S] \text {, and } \operatorname{rng} g \subset \underset{t}{E}[0 \leqq t \leqq \infty] \text {. }
$$

If $g$ is a gauge over $S, r>0$, define the function $\dot{g}_{r}$ for $x \subset S$ by the formula

$$
\dot{g}_{r}(x)=\inf _{G \in B} \sum_{y \in G} g(y),
$$

where $G \in B$ if and only if $G$ is such a countable family that $x \subset \sigma G$ and diam $y \leqq r$ whenever $y \in G$.

We shall say that $\phi$ is generated by $g$ if and only if $g$ is a gauge (over $S$ ) and $\phi$ is the function on

$$
\underset{x}{E}[x \subset S]
$$

defined by

$$
\phi(x)=\lim _{r \rightarrow 0+} \dot{g}_{r}(x) \quad \text { for } x \subset S .
$$

It is easy to check that $\phi$ is such a measure that closed sets are $\phi$ measurable.

2.9 Definition. Suppose $1 \leqq k \leqq n$. Let $g_{1}, g_{2}, g_{3}, g_{4}$ be the gauges over $E^{n}$ such that

dmn $g_{1}$ is the set of all open spheres of $E^{n}$,

dmn $g_{2}$ is the set of all subsets of $E^{n}$,

$g_{i}(x)=(k !)^{-1} \cdot \Gamma(1 / 2)^{k-1} \cdot \Gamma((k+1) / 2) \cdot(\operatorname{diam} x)^{k} \quad$ for $x \in \operatorname{dmn} g_{i}, i=1,2$, dmn $g_{3}$ is the set of all a nalytic subsets of $E^{n}$,

$g_{3}(x)=\beta(n, k)^{-1} \int_{G_{n}} \mathcal{L}_{k}\left[\left(p_{n}^{k}: R\right)^{*}(x)\right] d \phi_{n} R \quad$ for $x \in \operatorname{dmn} g_{8}$,

$\operatorname{dmn} g_{4}$ is the set of all convex open subsets of $E^{n}$,

$$
g_{4}(x)=\sup _{R \in G_{n}} \mathcal{L}_{k}\left[\left(p_{n}^{k}: R\right)^{*}(x)\right] \quad \text { for } x \in \operatorname{dmn} g_{4} .
$$

Then we define

$S_{n}^{k}$ is the measure generated by $g_{1}$, 
$\mathcal{F C}_{n}^{k}$ is the measure generated by $g_{2}$,

$\mathcal{F}_{n}^{k}$ is the measure generated by $g_{3}$,

$C_{n}^{k}$ is the measure generated by $g_{4}$.

$S_{n}^{k}$ is sphere $k$ measure over $n$ space, $\mathcal{F}_{n}^{\mathbf{k}}$ is Hausdorff measure, $\mathcal{F}_{n}^{k}$ is integralgeometric (Favard) measure, and $C_{n}^{k}$ is Carathéodory measure. We also define $\mathcal{F}_{n}^{0}$ by the formula

$$
\mathcal{F}_{n}^{0}(A)=\gamma(A)
$$

for $A \subset E^{n}$.

2.10 REMARK. It is quite easy to see that $S_{n}^{k}, \mathcal{F}_{n}^{k}, \mathcal{F}_{n}^{k}$, and $C_{n}^{k}$ are invariant under rigid motions of $E^{n}$, and that they satisfy the condition that any subset of $E^{n}$ is contained in an analytic set of the same measure.

We shall use the fact that if $A$ is an analytic subset of $E^{n}, 1 \leqq k<n$, then

$$
\begin{aligned}
\mathcal{F}_{n}^{k}(A) & =\beta(n, k)^{-1} \int_{G_{n}} \int_{E^{k}} N\left[\left(p_{n}^{k}: R\right), A, y\right] d \mathcal{L}_{k} y d \phi_{n} R \\
& =\beta(n, k)^{-1} \int_{G_{n}} \int_{E^{k}} \gamma\left[A \cap \lambda_{n}^{n-k}(R, y)\right] d \mathcal{L}_{k} y d \phi_{n} R .
\end{aligned}
$$

(See F2, 5.11] and [F3, 5].) Also it is obvious from 2.9 that

$$
\mathcal{F}_{n}^{n}=\mathcal{L}_{n}
$$

2.11 Definition. If $f$ is a function on a subset of $E^{k}$ to $E^{n}$, then $f$ is said to be Lipschitzian if and only if there is a number $M$ such that

$$
|f(x)-f(y)| \leqq M \cdot|x-y| \quad \text { for all } x, y \in \operatorname{dmn} f .
$$

We shall make use of the fact that if $f$ is Lipschitzian, $\operatorname{dmn} f \subset E^{k}, \operatorname{rng} f \subset E^{n}$, then there exists a Lipschitzian function $g$ on $E^{k}$ to $E^{n}$ such that $(g \mid \operatorname{dmn} f)=f$.

Suppose $1 \leqq k \leqq n, A \subset E^{n}$. Then we shall say that $A$ is $k$ rectifiable if and only if there is a Lipschitzian function whose domain is a bounded subset of $E^{k}$ and whose range is $A$.

3. A new formula involving Favard measure. Though Theorem 3.1 is not used in the remainder of this paper, I feel that it will be useful in future investigations concerning Favard measure.

3.1 Theorem. If $A$ is an analytic subset of $E^{n}, m$ and $k$ are such nonnegative integers that $k \leqq m \leqq n$, then

$$
\mathcal{F}_{n}^{k}(A)=(\beta(m, k) / \beta(n, k)) \cdot \int_{G_{n}} \int_{E^{m}} N\left[\left(p_{n}^{m}: R\right), A, y\right] d \mathcal{F}_{m}^{k} y d \phi_{n} R .
$$

Proof. With each $S \in G_{m}$, we associate the orthogonal transformation $\bar{S} \in G_{n}$ by the formulas 


$$
\begin{aligned}
& \bar{S}_{j}^{i}=S_{j}^{i} \\
& \bar{S}_{j}^{i}=0 \\
& \bar{S}_{j}^{i}=\delta_{j}^{i}
\end{aligned}
$$

$$
\begin{array}{r}
\text { for } i=1, \cdots, m ; \quad j=1, \cdots, m, \\
\text { for } i=1, \cdots, m ; \quad j=m+1, \cdots, n, \\
\text { for } i=m+1, \cdots, n ; j=1, \cdots, n,
\end{array}
$$

where $\delta_{j}^{t}$ is the ordinary Kronecker $\delta$-function.

The proof is divided into 3 parts.

Part 1. $\left(p_{m}^{k}: S: p_{n}^{m}: R\right)=\left(p_{n}^{k}: \bar{S}: R\right)$ for $R \in G_{n}, S \in G_{m}$.

Proof. For $x \in E^{n}$,

$$
\begin{aligned}
\left(p_{m}^{k}: S: p_{n}^{m}: R\right)(x) & =\left(p_{m}^{k}: S: p_{n}^{m}\right)\left(\sum_{i=1}^{n} x_{i} \cdot R^{i}\right) \\
& =p_{m}^{k}\left(\sum_{j=1}^{m}\left(\sum_{i=1}^{n} x_{i} \cdot R_{j}^{i}\right) S^{j}\right) \\
& =\sum_{h=1}^{k}\left(\sum_{j=1}^{m} \sum_{i=1}^{n} x_{i} R_{j}^{i} S_{h}^{j}\right){ }^{k} I^{h} \\
& =\sum_{h=1}^{k}\left(\sum_{j=1}^{n} \sum_{i=1}^{n} x_{i} R_{j}^{i} \bar{S}_{h}^{j}\right){ }^{k} I^{h} \\
& =\left(p_{n}^{k}: \bar{S}: R\right)(x) .
\end{aligned}
$$

This proves Part 1.

Part 2. For $R \in G_{n}$,

$\int_{E^{m}} N\left[\left(p_{n}^{m}: R\right), A, y\right] d \mathcal{F}_{m}^{k} y=\beta(m, k)^{-1} \int_{G_{m}} \int_{E^{k}} N\left[\left(p_{n}^{k}: \bar{S}: R\right), A, x\right] d \mathcal{L}_{k} x d \phi_{m} S$.

Proof. Let

$$
\begin{aligned}
& A_{i}=E^{m} \cap \underset{y}{E}\left[N\left[\left(p_{n}^{m}: R\right), A, y\right]=i\right] \quad \text { for } i=1,2, \cdots, \\
& A_{\infty}=E^{m} \cap \underset{y}{E}\left[N\left[\left(p_{n}^{m}: R\right), A, y\right]=\infty\right] .
\end{aligned}
$$

Then $A_{\infty}$ and $A_{i}$, for $i=1,2, \cdots$, are analytic and hence $\mathcal{F}_{m}^{k}$ measurable sets, and

$$
A_{i} \cap A_{i}=\varnothing \quad \text { for } i \neq j ; 1 \leqq i, j \leqq \infty
$$

Therefore

$$
\begin{aligned}
\int_{E^{m}} N\left[\left(p_{n}^{m}: R\right), A, y\right] d \mathcal{F}_{m}^{k} y & \\
& =\sum_{i=1}^{\infty} i \cdot \mathcal{F}_{m}^{k}\left(A_{i}\right)+\infty \cdot \mathcal{F}_{m}^{k}\left(A_{\infty}\right)
\end{aligned}
$$




$$
\begin{aligned}
= & \sum_{i=1}^{\infty} i \cdot \beta(m, k)^{-1} \int_{G_{m}} \int_{E^{k}} N\left[\left(p_{m}^{k}: S\right), A_{i}, x\right] d \mathcal{L}_{k} x d \phi_{m} S \\
& +\infty \cdot \beta(m, k)^{-1} \int_{G_{m}} \int_{E^{k}} N\left[\left(p_{m}^{k}: S\right), A_{\infty}, x\right] d \mathcal{L}_{k} x d \phi_{m} S \\
= & \beta(m, k)^{-1} \int_{G_{m}} \int_{E^{k}}\left(\sum_{i=1}^{\infty} i \cdot N\left[\left(p_{m}^{k}: S\right), A_{i}, x\right]\right. \\
& \left.+\infty \cdot N\left[\left(p_{m}^{k}: S\right), A_{\infty}, x\right]\right) d \mathcal{L}_{k} x d \phi_{m} S \\
= & \beta(m, k)^{-1} \int_{G_{m}} \int_{E^{k}} N\left[\left(p_{m}^{k}: S: p_{n}^{m}: R\right), A, x\right] d \mathcal{L}_{k} x d \phi_{m} S \\
= & \beta(m, k)^{-1} \int_{G_{m}} \int_{E^{k}} N\left[\left(p_{n}^{k}: \bar{S}: R\right), A, x\right] d \mathcal{L}_{k} x d \phi_{m} S .
\end{aligned}
$$

This completes the proof of Part 2.

Part 3.

$$
\mathcal{F}_{n}^{k}(A)=(\beta(m, k) / \beta(n, k)) \int_{G_{n}} \int_{E^{m}} N\left[\left(p_{n}^{m}: R\right), A, y\right] d \mathcal{F}_{m}^{k} y d \phi_{n} R .
$$

Proof. Applying Part 2 and 2.5,

$$
\begin{aligned}
\beta(m, k) \cdot \int_{G_{n}} \int_{E^{m}} N\left[\left(p_{n}^{m}: R\right),\right. & A, y] d \mathcal{F}_{m}^{k} y d \phi_{n} R \\
& =\int_{G_{n}} \int_{G_{m}} \int_{E^{k}} N\left[\left(p_{n}^{k}: \bar{S}: R\right), A, x\right] d \mathcal{L}_{k} x d \phi_{m} S d \phi_{n} R \\
& =\int_{G_{m}} \int_{G_{n}} \int_{E^{k}} N\left[\left(p_{n}^{k}: \bar{S}: R\right), A, x\right] d \mathcal{L}_{k} x d \phi_{n} R d \phi_{m} S \\
& =\int_{G_{m}} \int_{G_{n}} \int_{E^{k}} N\left[\left(p_{n}^{k}: R\right), A, x\right] d \mathcal{L}_{k} x d \phi_{n} R d \phi_{m} S \\
& =\int_{G_{n}} \int_{E^{k}} N\left[\left(p_{n}^{k}: R\right), A, x\right] d \mathcal{L}_{k} x d \phi_{n} R \\
& =\beta(n, k) \cdot \mathcal{F}_{n}^{k}(A) .
\end{aligned}
$$

This completes the proof.

4. The integralgeometric (Favard) measure of cylinder sets. In this section we consider the case in which $\beta=k=1$.

4.1 LEMMA. If

$$
1 \leqq i \leqq n,
$$




$$
\begin{aligned}
& S=\left(E^{n} \cap \underset{x}{E}[|x|=1]\right), \\
& \psi \text { is the function on } \underset{x}{E}[x \subset S] \text { defined by } \\
& \psi(x)=\phi_{n}\left(G_{n} \cap \underset{R}{E}\left[R^{i} \in X\right]\right)
\end{aligned}
$$

then

$$
\begin{aligned}
\psi(X) & =\left(\mathcal{H C}_{n}^{n-1}(S)\right)^{-1} \cdot \mathcal{H C}_{n}^{n-1}(X) \text { whenever } X \subset S \text { is a Borel set, } \\
\int_{G_{n}} g\left(R^{i}\right) d \phi_{n} R & =\left(\mathcal{F C}_{n}^{n-1}(S)\right)^{-1} \cdot \int_{S} g(x) d \mathcal{F C}_{n}^{n-1} x,
\end{aligned}
$$

whenever $g$ is a continuous function on $S$.

Proof. By straightforward methods it is checked that

$\psi$ measures $S$,

Open sets in the relative topology on $S$ are $\psi$ measurable,

$\psi(S)=1$, on $S$,

$\psi(X)>0$ whenever $X$ is an open non-empty set in the relative topology

$\psi\left[Q^{*}(X)\right]=\psi(X)$ whenever $Q \in G_{n}, X \subset S$.

Hence $\psi$ is a Haar measure over $S$ with respect to the transitive group $G_{n}$ of isometries of $S$. Since

$$
\left(\mathcal{H C}_{n}^{n-1} \mid \underset{x}{E}[x \subset S]\right)
$$

is also a Haar measure over $S$ with respect to $G_{n}$, we conclude by the uniqueness of Haar measures (on Borel sets) that (4) holds. (5) is now easily checked.

4.2 LEMma. If $1 \leqq i, j \leqq n$ and $-1 \leqq t \leqq 1$, then

$$
\phi_{n}\left(G_{n} \cap \underset{R}{E}\left[R_{j}^{i}=t\right]\right)=0 .
$$

Proof. By 4.1,

$$
\phi_{n}\left(G_{n} \cap \underset{R}{E}\left[R_{j}^{i}=t\right]\right)=\left(\mathcal{F C}_{n}^{n-1}(S)\right)^{-1} \cdot \mathcal{F C}_{n}^{n-1}\left(S \cap \underset{x}{E}\left[x_{j}=t\right]\right) .
$$

Since

$$
\mathfrak{H C}_{n}^{n-1}\left(S \cap \underset{x}{E}\left[x_{j}=t\right]\right)=0
$$

the proof is complete.

4.3 LEMMA. If $n$ is a positive integer, and $L$ is the set of those elements $R$ of $G_{n}$ for which ${ }^{n} I^{n}$ is a linear combination of $R^{2}, \cdots, R^{n}$, then $\phi_{n}(L)=0$. 
Proof. If $R \in L$, then $R^{1} \cdot{ }^{n} I^{n}=0$, and therefore $R_{n}^{1}=0$. Apply 4.2.

4.4 LEMMA. If $0 \leqq m<n-1$, then

$$
\phi_{n}\left(G_{n} \cap \underset{R}{E}\left[\sum_{i=m+2}^{n}\left|R_{n}^{i}\right|^{2}=1\right]\right)=0 .
$$

Proof. If $R \in G_{n}$ is such that $\sum_{i=m+2}^{n}\left|R_{n}^{i}\right|^{2}=1$, then in particular, $R_{u}^{1}=0$. Apply 4.2.

4.5 THEOREM. If

$$
\begin{aligned}
& 0 \leqq m \leqq n-1, \quad m \text { and } n \text { are integers, } \\
& A=E^{n} \cap \underset{x}{E}\left[x_{n}=0\right], \\
& B=E^{n} \cap \underset{x}{E}\left[x_{1}=x_{2}=\cdots=x_{n-1}=0\right], \\
& S \subset A, \quad S \text { is analytic, } \mathcal{F}_{n}^{m}(S)<\infty, \\
& T \subset B, \quad T \text { is analytic, } \mathcal{F}_{n}^{1}(T)<\infty,
\end{aligned}
$$

then

$$
\mathcal{F}_{n}^{m+1}(S+T)=\mathcal{F}_{n}^{1}(T) \cdot \mathcal{F}_{n}^{m}(S) .
$$

Proof. If $m=0$, let $q=\mathcal{F}_{n}^{0}(S)<\infty$. Then $S$ consists of $q$ distinct points of $A$. Therefore $(S+T)$ consists of $q$ distinct translates of $T$. Hence

$$
\mathcal{F}_{n}^{1}(S+T)=q \cdot \mathcal{F}_{n}^{1}(T)=\mathcal{F}_{n}^{0}(S) \cdot \mathcal{F}_{n}^{1}(T) \text {. }
$$

If $m=n-1$, let $S^{\prime}=\left(p_{n}^{n-1}\right)^{*}(S)$. Since $\eta_{n}^{n-1}$ is an isometric mapping of $S^{\prime}$ onto $S$, we use $[\mathrm{F} 1,4.5]$ to conclude that $\mathcal{F}_{n}^{n-1}(S)=\mathcal{L}_{n-1}\left(S^{\prime}\right)$. Similarly, if

$$
T^{\prime}=E^{1} \cap \underset{x}{E}[(0,0, \cdots, 0, x) \in T],
$$

then $\mathcal{F}_{n}^{1}(T)=\mathcal{L}_{1}\left(T^{\prime}\right)$. Thus by Fubini's theorem,

$$
\mathcal{F}_{n}^{n}(S+T)=\mathcal{L}_{n}(S+T)=\mathcal{L}_{n-1}\left(S^{\prime}\right) \cdot \mathcal{L}_{1}\left(T^{\prime}\right)=\mathcal{F}_{n}^{n-1}(S) \cdot \mathcal{F}_{n}^{1}(T) .
$$

Assume now that $0<m<n-1$. Without loss of generality we may assume that $\mathcal{F}_{n}^{1}(T)>0$. Let $K$ be the set of those elements $R$ of $G_{n}$ for which

$$
\sum_{i=m+2}^{n}\left|R_{n}^{i}\right|^{2}<1
$$

and for which $R^{2}, R^{3}, \cdots, R^{n},{ }^{n} I^{n}$ are linearly independent. Then by 4.3 and 4.4,

$$
\phi_{n}\left(G_{n}-K\right)=0 .
$$

Also it is easily seen that for $R \in K$, 


$$
\eta_{n}^{n-1}\left(p_{n}^{n-1}\left(R^{2}\right)\right), \eta_{n}^{n-1}\left(p_{n}^{n-1}\left(R^{3}\right)\right), \cdots, \eta_{n}^{n-1}\left(p_{n}^{n-1}\left(R^{n}\right)\right),
$$

are linearly independent. Hence, for each $R \in K$, we may consider orthogonal transformations which leave ${ }^{n} I^{n}$ fixed and which rotate ${ }^{n} I^{m+1}, \ldots,{ }^{n} I^{n-1}$ in such a way that they span the linear subspace generated by $\eta_{n}^{n-1}\left(p_{n}^{n-1}\left(R^{m+2}\right)\right)$, $\cdots, \eta_{n}^{n-1}\left(p_{n}^{n-1}\left(R^{n}\right)\right)$. For this purpose, we shall say that $P$ satisfies condition I with respect to $R$ if and only if $R \in K, P \in G_{n}$, and

$$
\begin{aligned}
& P\left({ }^{n} I^{n}\right)={ }^{n} I^{n}, \\
& P\left({ }^{n} I^{n-1}\right)=\eta_{n}^{n-1}\left(p_{n}^{n-1}\left(R^{n}\right)\right) /\left|\eta_{n}^{n-1}\left(p_{n}^{n-1}\left(R^{n}\right)\right)\right|, \\
& \left(^{*}\right) P\left(\frac{-R_{n}^{i} \cdot R_{n}^{n}}{\left(1-\left|R_{n}^{i}\right|^{2}\right)^{1 / 2}\left(1-\left|R_{n}^{n}\right|^{2}\right)^{1 / 2}} \cdot I^{n-1}+\right. \\
& \sum_{\alpha=0}^{n-i-2} \frac{-R_{n}^{i+1+\alpha} \cdot R_{n}^{i} \cdot{ }^{n} I^{i+\alpha}}{\left(1-\left|R_{n}^{i}\right|^{2}\right)^{1 / 2}\left(1-\sum_{j=1}^{n-i-1-\alpha}\left|R_{n}^{i+1+\alpha+j}\right|^{2}\right)^{1 / 2}\left(1-\sum_{j=0}^{n-i-1-\alpha}\left|R_{n}^{i+1+\alpha+j}\right|^{2}\right)^{1 / 2}} \\
& \left.+{ }^{n} I^{i-1} \frac{\left(1-\sum_{j=0}^{n-i}\left|R_{n}^{i+j}\right|^{2}\right)^{1 / 2}}{\left(1-\left|R_{n}^{i}\right|^{2}\right)^{1 / 2}\left(1-\sum_{j=1}^{n-i}\left|R_{n}^{i+j}\right|^{2}\right)^{1 / 2}}\right)=\frac{\eta_{n}^{n-1}\left(p_{n}^{n-1}\left(R^{i}\right)\right)}{\left|\eta_{n}^{n-1}\left(p_{n}^{n-1}\left(R^{i}\right)\right)\right|}
\end{aligned}
$$

for $i=m+2, \cdots, n-1$.

Further, we shall say that $Q$ satisfies condition II with respect to $R$ if and only if $R \in K, Q \in G_{n-1}$, and there is a $P \in G_{n}$ which satisfies condition I with respect to $R$ and for which

$$
Q_{j}^{i}=P_{j}^{i} \quad \text { for } i, j=1, \cdots, n-1 .
$$

In particular, we define the function $f$ on $K$ to $G_{n-1}$ such that if $R \in K$, then

$$
(f(R))_{j}^{i}=P_{i}^{i} \quad \text { for } i, j=1, \cdots, n-1,
$$

where $P$ satisfies condition I with respect to $R$ and also $P$ satisfies equation $\left.{ }^{*}\right)$ for $i=2, \cdots, n-1$. It is obvious that $f$ is a continuous mapping associating with each $R \in K$ a transformation $f(R)$ which satisfies condition II with respect to $R$.

Suppose $R \in K$ and $P$ satisfies condition I with respect to $R$. We shall reduce $P$ to a canonical form. Define $U_{P, R}=\left(P^{-1}: R\right)$. Then for $i=1,2, \cdots, m$, we have 


$$
{ }^{n} I^{i} \in U_{P, R}^{*}\left(E^{n} \cap \underset{x}{E}\left[x_{m+2}=\cdots=x_{n}=0\right]\right),
$$

because $\left(U_{P, R}\right)_{i}^{j}=0$ for $j=m+2, \cdots, n, i=1, \cdots, m$, and hence

$$
\left(U_{P, R}\right)^{i} \cdot{ }^{n} I^{i}=0 .
$$

We may therefore choose $\tilde{U}_{P, R} \in G_{m+1}$ such that

$$
\tilde{U}_{P, R}\left(\left(U_{P, R}^{-1}\left({ }^{n} I^{i}\right)\right)_{1}, \cdots,\left(U_{P, R}^{-1}\left(^{n} I^{i}\right)\right)_{m+1}\right)={ }^{m+1} I^{i} \quad \text { for } i=1, \cdots, m .
$$

Define $V_{P, R} \in G_{n}$ by the formulas

$$
\begin{array}{lr}
\left.V_{P, R}{ }^{n} I^{i}\right)=\left(\left(\tilde{U}_{P, R}\right)_{1}^{i}, \cdots,\left(\tilde{U}_{P, R}\right)_{m+1}^{i}, 0, \cdots, 0\right) & \text { for } i=1, \cdots, m+1, \\
V_{P, R}\left({ }^{n} I^{i}\right)={ }^{n} I^{i} & \text { for } i=m+2, \cdots, n .
\end{array}
$$

Finally, let $W_{P, R}=\left(U_{P, R}: V_{P, R}^{-1}\right) \in G_{n}$. Then it is easily checked that

$$
\begin{array}{rlrl}
W_{P, R}\left({ }^{n} I^{i}\right) & ={ }^{n} I^{i} & \text { for } i=1, \cdots, m, \\
\left.W_{P, R}{ }^{n} I^{i}\right) & \left.=U_{P, R}{ }^{n} I^{i}\right)=\left(P^{-1}: R\right)\left({ }^{n} I^{i}\right) & \text { for } i=m+2, \cdots, n, \\
\left(W_{P, R}\right)_{n}^{i} & =R_{n}^{i} & & \text { for } i=m+2, \cdots, n .
\end{array}
$$

The remainder of the proof is divided into 8 parts.

Part 1. If $P$ satisfies condition I with respect to $R$, then

$$
\begin{aligned}
\int_{E^{m+1}} & \gamma\left[(S+T) \cap \lambda_{n}^{n-m-1}(R, y)\right] d \mathcal{L}_{m+1} y \\
\quad & \int_{E^{m}} \int_{E^{1}} \gamma\left[\left(P^{-1 *}(S)+T\right) \cap \lambda_{n}^{n-m-1}\left(W_{P, R},\left(x_{1}, \cdots, x_{m}, y\right)\right)\right] d \mathcal{L}_{1} y d \mathcal{L}_{m} x .
\end{aligned}
$$

Proof. Since $\mathcal{L}_{m+1}$ is invariant under transformations by elements of $G_{m+1}$, and applying Fubini's Theorem, we have

$$
\begin{aligned}
\int_{E^{m+1}} & \gamma\left[(S+T) \cap \lambda_{n}^{n-m-1}(R, y)\right] d \mathcal{L}_{m+1} y \\
= & \int_{E^{m+1}} \gamma\left[P^{-1 *}\left((S+T) \cap \lambda_{n}^{n-m-1}(R, y)\right)\right] d \mathcal{L}_{m+1} y \\
= & \left.\int_{E^{m+1}} \gamma\left[P^{-1 *}(S)+T\right) \cap \lambda_{n}^{n-m-1}\left(P^{-1}: R, y\right)\right] d \mathcal{L}_{m+1} y \\
= & \int_{E^{m+1}} \gamma\left[\left(P^{-1 *}(S)+T\right) \cap \lambda_{n}^{n-m-1}\left(U_{P, R}, \tilde{U}_{P, R}^{-1}(y)\right)\right] d \mathcal{L}_{m+1} y \\
= & \int_{E^{m+1}} \gamma\left[\left(P^{-1 *}(S)+T\right) \cap \lambda_{n}^{n-m-1}\left(\left(U_{P, R}: V_{P, R}^{-1}\right), y\right)\right] d \mathcal{L}_{m+1} y
\end{aligned}
$$


$=\int_{E^{m}} \int_{E^{1}} \gamma\left[\left(P^{-1 *}(S)+T\right) \cap \lambda_{n}^{n-m-1}\left(W_{P, R},\left(x_{1}, \cdots, x_{m}, y\right)\right)\right] d \mathcal{L}_{1} y d \mathcal{L}_{m} x$.

This completes Part 1.

Part 2. If $P$ satisfies condition I with respect to $R$, then

$$
\left|\left(W_{P, R}\right)_{n}^{m+1}\right|=\left(\sum_{j=1}^{m+1}\left|R_{n}^{j}\right|^{2}\right)^{1 / 2}>0 .
$$

Proof. Since $P$ and $R$ are fixed, we omit the subscripts on $W_{P, R}$. Notice that

$$
\begin{aligned}
& 0=W^{m+1} \cdot W^{n}=W_{n-1}^{m+1}\left(1-\left|R_{n}^{n}\right|^{2}\right)^{1 / 2}+W_{n}^{m+1} R_{n}^{n}, \\
& 0=W^{m+1} \cdot W^{n-1}=W_{n-2}^{m+1} \cdot \frac{\left(1-\left|R_{n}^{n-1}\right|^{2}-\left|R_{n}^{n}\right|^{2}\right)^{1 / 2}}{\left(1-\left|R_{n}^{n}\right|^{2}\right)^{1 / 2}}-W_{n-1}^{m+1} \cdot \frac{R_{n}^{n-1} \cdot R_{n}^{n}}{\left(1-\left|R_{n}^{n}\right|^{2}\right)^{1 / 2}} \\
& +W_{n}^{m+1} R_{n}^{n-1} \\
& 0=W^{m+1} \cdot W^{m+2}=W_{m+1}^{m+1} \frac{\left(1-\sum_{j=m+2}^{n}\left|R_{n}^{j}\right|^{2}\right)^{1 / 2}}{\left(1-\sum_{j=m+3}^{n}\left|R_{n}^{i}\right|^{2}\right)^{1 / 2}}-\sum_{\alpha=m+2}^{n-2} W_{\alpha}^{m+1} \\
& \frac{R_{n}^{\alpha+1} \cdot R_{n}^{m+2}}{\left(1-\sum_{j=1}^{(n-\alpha-1)}\left|R_{n}^{\alpha+1+j}\right|^{2}\right)^{1 / 2} \cdot\left(1-\sum_{j=0}^{(n-\alpha-1)}\left|R_{n}^{\alpha+1+j}\right|^{2}\right)^{1 / 2}}-W_{n-1}^{m+1} \\
& \frac{R_{n}^{m+2} \cdot R_{n}^{n}}{\left(1-\left|R_{n}^{n}\right|^{2}\right)^{1 / 2}}+W_{n}^{m+1} R_{n}^{m+2} \text {, } \\
& 1=W^{m+1} \cdot W^{m+1}=\sum_{\alpha=m+1}^{n}\left|W_{\alpha}^{m+1}\right|^{2} \text {. }
\end{aligned}
$$

Hence solving for $\left|W_{n}^{m+1}\right|$, we have

$$
\begin{aligned}
& W_{n-1}^{m+1}=-\frac{R_{n}^{n}}{\left(1-\left|R_{n}^{n}\right|^{2}\right)^{1 / 2}} \cdot W_{n}^{m+1}, \\
& W_{m+1}^{m+1}=-\frac{R_{n}^{m+2}}{\left(1-\sum_{j=m+3}^{n}\left|R_{n}^{j}\right|^{2}\right)^{1 / 2}\left(1-\sum_{j=m+2}^{n}\left|R_{n}^{j}\right|^{2}\right)^{1 / 2}} W_{n}^{m+1},
\end{aligned}
$$


and therefore

$$
\begin{aligned}
\left|W_{n}^{m+1}\right|^{2} \cdot(1 & +\frac{\left|R_{n}^{n}\right|^{2}}{1-\left|R_{n}^{n}\right|^{2}}+\frac{\left|R_{n}^{n-1}\right|^{2}}{\left(1-\left|R_{n}^{n}\right|^{2}\right)\left(1-\left|R_{n}^{n-1}\right|^{2}-\left|R_{n}^{n}\right|^{2}\right)}+\cdots \\
& \left.+\frac{\left|R_{n}^{m+2}\right|^{2}}{\left(1-\sum_{j=m+3}^{n}\left|R_{n}^{j}\right|^{2}\right)\left(1-\sum_{j=m+2}^{n}\left|R_{n}^{j}\right|^{2}\right)}\right) \\
= & \left|W_{n}^{m+1}\right|^{2} \cdot\left(\frac{1}{1-\sum_{j=m+2}^{n}\left|R_{n}^{j}\right|^{2}}\right)=1, \\
\left|W_{n}^{m+1}\right|= & \left(\sum_{j=1}^{m+1}\left|R_{n}^{j}\right|^{2}\right)^{1 / 2}>0 .
\end{aligned}
$$

The proof of Part 2 is complete.

Part 3. If $P$ satisfies condition I with respect to $R$, then

$$
\begin{aligned}
\mathcal{L}_{1}\left(\underset { y } { E } \left[\left(\left(\left\{\theta^{n}\right\}+T\right) \cap \lambda_{n}^{n-m-1}\left(W_{P, R},(0, \cdots, 0, y)\right)\right)\right.\right. & \neq \varnothing]) \\
& =\left(\sum_{j=1}^{m+1}\left|R_{n}^{j}\right|^{2}\right)^{1 / 2} \cdot \mathcal{F}_{n}^{1}(T)>0 .
\end{aligned}
$$

Proof. Let

$$
T^{\prime}=E^{1} \cap \underset{x}{E}[(0, \cdots, 0, x) \in T]
$$

Recalling that

$$
\begin{array}{rrr}
\left(W_{P, R}\right)^{m+1} \cdot\left(W_{P, R}\right)^{i}=0 & \text { for } i \neq m+1, \\
\left(W_{P, R}\right)_{j}^{m+1}=0 & \text { for } j=1, \cdots, m,
\end{array}
$$

we have

$$
\begin{aligned}
& {\left[\left(\left(\left\{\theta^{n}\right\}+T\right) \cap \lambda_{n}^{n-m-1}\left(W_{P, R},(0, \cdots, 0, y)\right)\right) \neq \varnothing\right]} \\
& \quad \rightleftarrows\left\{\begin{array}{l}
\text { There are numbers } \alpha_{m+2}, \cdots, \alpha_{n} \text { such that } \\
y \cdot\left(W_{P, R}\right)_{j}^{m+1}+\sum_{i=m+2}^{n} \alpha_{i} \cdot\left(W_{P, R}\right)_{j}^{i}=0 \\
y \cdot\left(W_{P, R}\right)_{n}^{m+1}+\sum_{i=m+2}^{n} \alpha_{i} \cdot\left(W_{P, R}\right)_{n}^{i} \in T^{\prime},
\end{array} \text { for } j=m+1, \cdots, n-1,\right.
\end{aligned}
$$

which implies that 
$y \cdot \sum_{j=m+1}^{n}\left|\left(W_{P, R}\right)_{j}^{m+1}\right|^{2}+\sum_{i=m+2}^{n} \alpha_{i} \cdot \sum_{j=m+1}^{n}\left(W_{P, R}\right)_{j}^{m+1} \cdot\left(W_{P, R}\right)_{j}^{i}=y \in\left(W_{P, R}\right)_{n}^{m+1} \cdot T^{\prime}$, where $\left(W_{P, R}\right)_{n}^{m+1} \cdot T^{\prime}$ shall mean

$$
\left(E^{1} \cap \underset{x}{E}\left[x=\left(W_{P, R_{n}}\right)_{n}^{m+1} \cdot y \text { for some } y \in T^{\prime}\right] .\right.
$$

Conversely, if $y \in\left(W_{P, R}\right)_{n}^{m+1} \cdot T^{\prime}$, let

$$
\begin{aligned}
& \alpha_{m+2}=-\frac{y \cdot\left(W_{P, R}\right)_{m+1}^{m+1}}{\left(W_{P, R}\right)_{m+1}^{m+2}} \\
& \alpha_{m+3}=-\frac{y \cdot\left(W_{P, R}\right)_{m+2}^{m+1}+\alpha_{m+2} \cdot\left(W_{P, R}\right)_{m+2}^{m+2}}{\left(W_{P, R}\right)_{m+2}^{m+3}} \\
& \alpha_{n}=-\frac{y \cdot\left(W_{P, R}\right)_{n-1}^{m+1}+\alpha_{m+2} \cdot\left(W_{P, R}\right)_{n-1}^{m+2}+\cdots+\alpha_{n-1}\left(W_{P, R}\right)_{n-1}^{n-1}}{\left(W_{P, R}\right)_{n-1}^{n}} .
\end{aligned}
$$

Then it easily follows that

$$
y \cdot\left(W_{P, R}\right)_{n}^{m+1}+\sum_{i=m+2}^{n} \alpha_{i} \cdot\left(W_{P, R}\right)_{n}^{i} \in T^{\prime} .
$$

Hence, by Part 2 and the fact that $\mathcal{L}_{1}\left(T^{\prime}\right)=\mathcal{F}_{n}^{1}(T)>0$,

$$
\begin{aligned}
& \mathcal{L}_{1}\left(\underset{y}{E}\left[\left(\left(\left\{\theta^{n}\right\}+T\right) \cap \lambda_{n}^{n-m-1}\left(W_{P, R},(0, \cdots, 0, y)\right)\right) \neq \varnothing\right]\right) \\
& \quad=\mathcal{L}_{1}\left(\left(W_{P, R}\right)_{n}^{m+1} \cdot T^{\prime}\right)=\left|\left(W_{P, R}\right)_{n}^{m+1}\right| \cdot \mathcal{L}_{1}\left(T^{\prime}\right)=\left(\sum_{j=1}^{m+1}\left|R_{n}^{j}\right|^{2}\right)^{1 / 2} \cdot \mathcal{F}_{n}^{1}(T)>0 .
\end{aligned}
$$

This completes Part 3.

Part 4. If $P$ satisfies condition I with respect to $R$, and $Q \in G_{n-1}$ is such that $Q_{j}^{i}=P_{j}^{i}$ for $i, j=1, \cdots, n-1$, then

$$
\begin{aligned}
\int_{E^{1}} \gamma\left[\left(P^{-1 *}(S)\right.\right. & \left.+T) \cap \lambda_{n}^{n-m-1}\left(W_{P, R},\left(x_{1}, \cdots, x_{m}, y\right)\right)\right] d \mathcal{L}_{1} y \\
= & \gamma\left[\left(Q^{-1}: p_{n}^{n-1}\right)^{*}(S) \cap \lambda_{n-1}^{n-m-1}\left({ }^{(n-1)} I, x\right)\right] \cdot\left(\sum_{j=1}^{m+1}\left|R_{n}^{j}\right|^{2}\right)^{1 / 2} \cdot \mathcal{F}_{n}^{1}(T) .
\end{aligned}
$$

Proof. Assume that

$$
q=\gamma\left[\left(Q^{-1}: p_{n}^{n-1}\right)^{*}(S) \cap \lambda_{n-1}^{n-m-1}\left({ }^{(n-1)} I, x\right)\right]<\infty .
$$


Then

$$
\left(\left(Q^{-1}: p_{n}^{n-1}\right)^{*}(S) \cap \lambda_{n-1}^{n-m-1}\left({ }^{(n-1)} I, x\right)\right)=\bigcup_{i=1}^{q}\left\{z^{i}\right\},
$$

where $z^{i} \neq z^{i}$ for $i \neq j$ and $z^{i} \in E^{n-1}$ for $i=1, \cdots, q$. Furthermore

$$
\begin{aligned}
\int_{E^{1}} \gamma[ & \left.\left(P^{-1 *}(S)+T\right) \cap \lambda_{n}^{n-m-1}\left(W_{P, R},\left(x_{1}, \cdots, x_{m}, y\right)\right)\right] d \mathcal{L}_{1} y \\
& =\int_{E^{1}} \sum_{i=1}^{q} \gamma\left[\left(\left\{\eta_{n}^{n-1}\left(z^{i}\right)\right\}+T\right) \cap \lambda_{n}^{n-m-1}\left(W_{P, R},\left(x_{1}, \cdots, x_{m}, y\right)\right)\right] d \mathcal{L}_{1} y \\
& =q \cdot \int_{E^{1}} \gamma\left[\left(\left\{\left(x_{1}, \cdots, x_{m}, 0, \cdots, 0\right)\right\}+T\right)\right. \\
& \left.\cap \lambda_{n}^{n-m-1}\left(W_{P, R},\left(x_{1}, \cdots, x_{m}, y\right)\right)\right] d \mathcal{L}_{1} y \\
& =q \cdot \int_{E^{1}} \gamma\left[\left(\left\{\theta^{n}\right\}+T\right) \cap \lambda_{n}^{n-m-1}\left(W_{P, R},(0, \cdots, 0, y)\right)\right] d \mathcal{L}_{1} y .
\end{aligned}
$$

Now if $(0, \cdots, 0, a)$ and $(0, \cdots, 0, b)$ are both in $\lambda_{n}^{m-n-1}\left(W_{P, R},(0, \cdots\right.$, $0, y)$ ), then there exist numbers $\alpha_{m+2}, \cdots, \alpha_{n}$, and $\beta_{m+2}, \cdots, \beta_{n}$ such that

$$
\begin{aligned}
& \left\{\begin{array}{l}
y \cdot\left(W_{P, R}\right)_{n}^{m+1}+\alpha_{m+2} \cdot\left(W_{P, R}\right)_{n}^{m+2}+\cdots+\alpha_{n} \cdot\left(W_{P, R}\right)_{n}^{n}=a, \\
y \cdot\left(W_{P, R}\right)_{n}^{m+1}+\beta_{m+2} \cdot\left(W_{P, R}\right)_{n}^{m+2}+\cdots+\beta_{n} \cdot\left(W_{P, R}\right)_{n}^{n}=b,
\end{array}\right. \\
& \left\{\begin{array}{l}
y \cdot\left(W_{P, R}\right)_{m+1}^{m+1}+\alpha_{m+2} \cdot\left(W_{P, R}\right)_{m+1}^{m+2}=0 \\
y \cdot\left(W_{P, R}\right)_{m+1}^{m+1}+\beta_{m+2} \cdot\left(W_{P, R}\right)_{m+1}^{m+2}=0
\end{array}\right. \\
& \left\{\begin{array}{l}
y \cdot\left(W_{P, R^{t}}\right)_{n-1}^{m+1}+\alpha_{m+2} \cdot\left(W_{P, R}\right)_{n-1}^{m+2}+\cdots+\alpha_{n} \cdot\left(W_{P, R}\right)_{n-1}^{n}=0, \\
y \cdot\left(W_{P, R^{\prime}}\right)_{n-1}^{m+1}+\beta_{m+2} \cdot\left(W_{P, R^{\prime}}\right)_{n-1}^{m+2}+\cdots+\beta_{n} \cdot\left(W_{P, R}\right)_{n-1}^{n}=0 .
\end{array}\right.
\end{aligned}
$$

From the definition of $W_{P, R}$ we see that

$$
\left(W_{P, R}\right)_{m+1+j}^{m+2+j} \neq 0 \quad \text { for } j=0, \cdots, n-m-2,
$$

hence

$$
\alpha_{m+2}=\beta_{m+2}, \cdots, \alpha_{n}=\beta_{n},
$$

and therefore $(0, \cdots, 0, a)=(0, \cdots, 0, b)$. From this it follows that

$$
\int_{E^{1}} \gamma\left[\left(\left\{\theta^{n}\right\}+T\right) \cap \lambda_{n}^{n-m-1}\left(W_{P, R},(0, \cdots, 0, y)\right)\right] d \mathcal{L}_{1} y
$$




$$
\begin{aligned}
& =\mathcal{L}_{1}\left(\underset{y}{E}\left[\left(\left(\left\{\theta^{n}\right\}+T\right) \cap \lambda_{n}^{n-m-1}\left(W_{P, R},(0, \cdots, 0, y)\right)\right) \neq \varnothing\right]\right) \\
& =\left(\sum_{j=1}^{m+1}\left|R_{n}^{j}\right|^{2}\right)^{1 / 2} \cdot \mathcal{F}_{n}^{1}(T)>0 .
\end{aligned}
$$

Hence

$$
\begin{aligned}
\int_{E^{1}} \gamma\left[\left(P^{-1 *}(S)+T\right) \cap \lambda_{n}^{n-m-1}\left(W_{P, R},\left(x_{1}, \cdots, x_{m}, y\right)\right)\right] d \mathcal{L}_{1} y & \\
& =q \cdot\left(\sum_{j=1}^{m+1}\left|R_{n}^{j}\right|^{2}\right)^{1 / 2} \cdot \mathcal{F}_{n}^{1}(T) .
\end{aligned}
$$

The same method handles the case $q=\infty$, since

$$
\left(\sum_{j=1}^{m+1}\left|R_{n}^{j}\right|^{2}\right)^{1 / 2} \cdot \mathcal{F}_{n}^{1}(T)>0
$$

This completes the proof of Part 4.

Part 5. If $Q$ satisfies condition II with respect to $R$, then

$$
\begin{aligned}
\int_{E^{m+1}} \gamma[(S+T) & \left.\cap \lambda_{n}^{n-m-1}(R, y)\right] d \mathcal{L}_{m+1} y \\
= & \left(\sum_{j=1}^{m+1}\left|R_{n}^{i}\right|^{2}\right)^{1 / 2} \cdot \mathcal{F}_{n}^{1}(T) \cdot \int_{E^{m}} \gamma\left[p_{n}^{n-1 *}(S) \cap \lambda_{n-1}^{n-m-1}(Q, x)\right] d \mathcal{L}_{m} x .
\end{aligned}
$$

Proof. Applying Parts 1 and 4, we have

$$
\begin{aligned}
\int_{E^{m+1}} & \gamma\left[(S+T) \cap \lambda_{n}^{n-m-1}(R, y)\right] d \mathcal{L}_{m+1} y \\
= & \int_{E^{m}}\left(\sum_{j=1}^{m+1}\left|R_{n}^{i}\right|^{2}\right)^{1 / 2} \cdot \mathcal{F}_{n}^{1}(T) \cdot \gamma\left[\left(Q^{-1}: p_{n}^{n-1}\right)^{*}(S) \cap \lambda_{n-1}^{n-m-1}\left({ }^{(n-1)} I, x\right)\right] d \mathcal{L}_{m} x \\
= & \left(\sum_{i=1}^{m+1}\left|R_{n}^{i}\right|^{2}\right)^{1 / 2} \cdot \mathcal{F}_{n}^{1}(T) \cdot \int_{E^{m}} \gamma\left[p_{n}^{n-1 *}(S) \cap \lambda_{n-1}^{n-m-1}(Q, x)\right] d \mathcal{L}_{m} x .
\end{aligned}
$$

The proof of Part 5 is complete.

For the remainder of this proof, we associate with each $P \in G_{n-1}$ the transformation $\widetilde{P} \in G_{n}$ by the formulas

$$
\begin{aligned}
& \tilde{P}^{i}=\eta_{n}^{n-1}\left(P^{i}\right) \quad \text { for } i=1, \cdots, n-1, \\
& \tilde{P}^{n}={ }^{n} I^{n} .
\end{aligned}
$$

Part 6. If $Q$ satisfies condition II with respect to $R$, and $P \in G_{n-1}$, then $(P: Q)$ satisfies condition II with respect to $(\tilde{P}: R)$.

Proof. We have for $1 \leqq i \leqq n-1,1 \leqq k \leqq n$, 


$$
(\widetilde{P}: R)_{i}^{k}=\sum_{j=1}^{n} R_{i}^{k} \cdot \tilde{P}_{i}^{j}=\sum_{j=1}^{n-1} R_{j}^{k} \cdot P_{i}^{i},
$$

and

$$
(\widetilde{P}: R)_{n}^{k}=\sum_{j=1}^{n} R_{j}^{k} \cdot \widetilde{P}_{n}^{j}=R_{n}^{k}
$$

Hence

$$
\begin{aligned}
& (P: Q)\left({ }^{(n-1)} I^{n-1}\right)=P\left(p_{n}^{n-1}\left(R^{n}\right) /\left|p_{n}^{n-1}\left(R^{n}\right)\right|\right) \\
& =\left(1-\left|R_{n}^{n}\right|^{2}\right)^{-1 / 2} \cdot P\left(R_{1}^{n}, \cdots, R_{n-1}^{n}\right) \\
& =\left(1-\left|R_{n}^{n}\right|^{2}\right)^{-1 / 2} \cdot \sum_{i=1}^{n-1} R_{i}^{n} \cdot P^{i} \\
& =\left(1-\left|(\widetilde{P}: R)_{n}^{n}\right|^{2}\right)^{-1 / 2}\left((\widetilde{P}: R)_{1}^{n}, \cdots,(\tilde{P}: R)_{n-1}^{n}\right) \\
& =p_{n}^{n-1}\left((\widetilde{P}: R)^{n}\right) /\left|p_{n}^{n-1}\left((\widetilde{P}: R)^{n}\right)\right| \text {, } \\
& (P: Q)\left(\frac{-(\tilde{P}: R)_{n}^{i} \cdot(\tilde{P}: R)_{n}^{n}}{\left(1-\left|(\tilde{P}: R)_{n}^{i}\right|^{2}\right)^{1 / 2}\left(1-\left|(\tilde{P}: R)_{n}^{n}\right|^{2}\right)^{1 / 2}} \cdot{ }^{(n-1)} I^{n-1}\right. \\
& +\sum_{\alpha=0}^{n-i-2} \frac{-(\widetilde{P}: R)_{n}^{i+1+\alpha} \cdot(\tilde{P}: R)_{n}^{i} \cdot{ }^{(n-1)} I^{i+\alpha}}{\left(1-\left|(\widetilde{P}: R)_{n}^{i}\right|^{2}\right)^{1 / 2}\left(1-\sum_{j=1}^{n-i-1-\alpha}\left|(\widetilde{P}: R)_{n}^{i+1+\alpha+i}\right|^{2}\right)^{1 / 2}} \\
& \overline{\left(1-\sum_{j=0}^{n-i-1-\alpha}\left|(\tilde{P}: R)_{n}^{i+1+\alpha+i}\right|^{2}\right)^{1 / 2}} \\
& \left(1-\sum_{j=0}^{n-i}\left|(\widetilde{P}: R)_{n}^{i+j}\right|^{2}\right)^{1 / 2} \\
& \left.+\frac{\left(1-\left|(\tilde{P}: R)_{n}^{i}\right|^{2}\right)^{1 / 2}\left(1-\sum_{j=1}^{n-i}\left|(\tilde{P}: R)_{n}^{i+i}\right|^{2}\right)^{1 / 2}}{(n-1)} I^{i-1}\right) \\
& =P\left(p_{n}^{n-1}\left(R^{i}\right) /\left|p_{n}^{n-1}\left(R^{i}\right)\right|\right) \\
& =\left(1-\left|R_{n}^{i}\right|^{2}\right)^{-1 / 2} \cdot \sum_{j=1}^{n-1} R_{j}^{i} P^{i} \\
& =\left(1-\left|(\tilde{P}: R)_{n}^{i}\right|^{2}\right)^{-1 / 2}\left((\tilde{P}: R)_{1}^{i}, \cdots,(\tilde{P}: R)_{n-1}^{i}\right) \\
& =p_{n}^{n-1}\left((\tilde{P}: R)^{i}\right) /\left|p_{n}^{n-1}\left((\tilde{P}: R)^{i}\right)\right| \text {, }
\end{aligned}
$$

for $i=m+2, \cdots, n-1$. 

$(\tilde{P}: R)$.

It easily follows now that $(P: Q)$ satisfies condition II with respect to

Part 7.

$$
\begin{aligned}
\mathcal{F}_{n}^{m+1}(S+T)= & \mathcal{F}_{n}^{m}(S) \cdot \mathcal{F}_{n}^{1}(T) \cdot(\beta(n-1, m) / \beta(n, m+1)) \\
& \cdot \int_{G_{n}}\left(\sum_{j=1}^{m+1}\left|R_{n}^{j}\right|^{2}\right)^{1 / 2} d \phi_{n} R .
\end{aligned}
$$

Proof. Recalling the definition of $f$, and using Parts 5 and 6, we have for $P \in G_{n-1}$

$\mathcal{F}_{n}^{m+1}(S+T)$

$$
\begin{aligned}
& =\beta(n, m+1)^{-1} \cdot \int_{G_{n}} \int_{E^{m+1}} \gamma\left[(S+T) \cap \lambda_{n}^{n-m-1}(R, y)\right] d \mathcal{L}_{m+1} y d \phi_{n} R \\
& =\beta(n, m+1)^{-1} \cdot \int_{K} \int_{E^{m+1}} \gamma\left[(S+T) \cap \lambda_{n}^{n-m-1}(R, y)\right] d \mathcal{L}_{m+1} y d \phi_{n} R \\
& =\beta(n, m+1)^{-1} \cdot \int_{K} \int_{E^{m+1}} \gamma\left[(S+T) \cap \lambda_{n}^{n-m-1}((\tilde{P}: R), y)\right] d \mathcal{L}_{m+1} y d \phi_{n} R \\
& =\beta(n, m+1)^{-1} \cdot \int_{K}\left(\sum_{j=1}^{m+1}\left|R_{n}^{j}\right|^{2}\right)^{1 / 2} \cdot \mathcal{F}_{n}^{1}(T) \\
& \cdot \int_{E^{m}} \gamma\left[p_{n}^{n-1 *}(S) \cap \lambda_{n-1}^{n-m-1}((P: f(R)), x)\right] d \mathcal{C}_{m} x d \phi_{n} R .
\end{aligned}
$$

Hence

$$
\begin{aligned}
& \mathcal{F}_{n}^{m+1}(S+T) \\
&=\mathcal{F}_{n}^{1}(T) \cdot(\beta(n, m+1))^{-1} \cdot \int_{G_{n-1}} \int_{K}\left(\sum_{j=1}^{m+1}\left|R_{n}^{j}\right|^{2}\right)^{1 / 2} \\
& \cdot \int_{E^{m}} \gamma\left[p_{n}^{n-1 *}(S) \cap \lambda_{n-1}^{n-m-1}((P: f(R)), x)\right] d \mathcal{L}_{m} x d \phi_{n} R d \phi_{n-1} P \\
&=\mathcal{F}_{n}^{1}(T) \cdot(\beta(n, m+1))^{-1} \cdot \int_{K}\left(\sum_{j=1}^{m+1}\left|R_{n}^{j}\right|^{2}\right)^{1 / 2} \\
& \cdot \int_{G_{n-1}} \int_{E^{m}} \gamma\left[p_{n}^{n-1 *}(S) \cap \lambda_{n-1}^{n-m-1}((P: f(R)), x)\right] d \mathcal{L}_{m} x d \phi_{n-1} P d \phi_{n} R \\
&= \beta(n-1, m)(\beta(n, m+1))^{-1} \cdot \mathcal{F}_{n}^{1}(T) \\
& \cdot \mathcal{F}_{n-1}^{m}\left(p_{n}^{n-1 *}(S)\right) \int_{K}\left(\sum_{j=1}^{m+1}\left|R_{n}^{j}\right|^{2}\right)^{1 / 2} d \phi_{n} R
\end{aligned}
$$




$$
\begin{aligned}
= & \mathcal{F}_{n}^{m}(S) \cdot \mathscr{F}_{n}^{1}(T) \cdot \beta(n-1, m) \cdot(\beta(n, m+1))^{-1} \\
& \cdot \int_{G_{n}}\left(\sum_{j=1}^{m+1}\left|R_{n}^{j}\right|^{2}\right)^{1 / 2} d \phi_{n} R .
\end{aligned}
$$

The interchange of the order of integration in the above is justified by the following argument:

The function $g$ defined on $G_{n-1}$ by

$$
g(P)=\int_{E^{m}} \gamma\left[p_{n}^{n-1 *}(S) \cap \lambda_{n-1}^{n-m-1}(P, x)\right] d \mathcal{L}_{m} x \quad \text { for } P \in G_{n-1},
$$

is such that counter-images of open sets are analytic sets. Using the fact that counter-images of analytic sets by continuous functions are again analytic sets, we conclude that the function $h$ on $\left(G_{n-1} \times K\right)$ is measurable, where

$$
h(P, R)=\int_{E^{m}} \gamma\left[p_{n}^{n-1 *}(S) \cap \lambda_{n-1}^{n-m-1}((P: f(R), x)] d \mathcal{L}_{m} x,\right.
$$

for $(P, R) \in\left(G_{n-1} \times K\right)$. The Fubini Theorem then applies immediately.

The proof of Part 7 is complete.

Part 8. $\mathcal{F}_{n}^{m+1}(S+T)=\mathcal{F}_{n}^{m}(S) \cdot \mathcal{F}_{n}^{1}(T)$.

Proof. To evaluate the factor

$$
\beta(n-1, m)(\beta(n, m+1))^{-1} \int_{G_{n}}\left(\sum_{j=1}^{m+1}\left|R_{n}^{i}\right|^{2}\right)^{1 / 2} d \phi_{n} R,
$$

we take in particular

$$
\begin{aligned}
& T_{0}=E^{n} \cap \underset{x}{E}\left[x_{1}=x_{2}=\cdots=x_{n-1}=0,1 \geqq x_{n} \geqq 0\right], \\
& S_{0}=E^{n} \cap \underset{x}{E}\left[0 \leqq x_{i} \leqq 1 \text { for } i=1, \cdots, m \text {, and } x_{m+1}=\cdots=x_{n}=0\right] .
\end{aligned}
$$

Then

$$
\mathcal{F}_{n}^{m+1}\left(S_{0}+T_{0}\right)=1=\mathcal{F}_{n}^{1}\left(T_{0}\right)=\mathcal{F}_{n}^{m}\left(S_{0}\right)
$$

therefore

$$
\beta(n, m+1)(\beta(n-1, m))^{-1}=\int_{G_{n}}\left(\sum_{j=1}^{m+1}\left|R_{n}^{j}\right|^{2}\right)^{1 / 2} d \phi_{n} R,
$$

and we conclude that

$$
\mathcal{F}_{n}^{m+1}(S+T)=\mathcal{F}_{n}^{m}(S) \cdot \mathcal{F}_{n}^{1}(T),
$$

for all $S, T$ satisfying the hypotheses of this theorem. 
4.6 Corollary. If $m+1 \leqq n$, then

$$
\beta(n, m+1)(\beta(n-1, m))^{-1}=\int_{G_{n}}\left(\sum_{j=1}^{m+1}\left|R_{j}^{n}\right|^{2}\right)^{1 / 2} d \phi_{n} R .
$$

Proof. Recall that for $R \in G_{n}$,

$$
\left(R^{-1}\right)_{j}^{i}=R_{i}^{j} \quad \text { for } 1 \leqq i, j \leqq n .
$$

Hence by 4.5 and 2.5 ,

$$
\begin{aligned}
\beta(n, m+1)(\beta(n-1, m))^{-1} & =\int_{G_{n}}\left(\sum_{j=1}^{m+1}\left|R_{n}^{j}\right|^{2}\right)^{1 / 2} d \phi_{n} R \\
& =\int_{G_{n}}\left(\sum_{j=1}^{m+1}\left|\left(R^{-1}\right)_{j}^{n}\right|^{2}\right)^{1 / 2} d \phi_{n} R \\
& =\int_{G_{n}}\left(\sum_{j=1}^{m+1}\left|R_{j}^{n}\right|^{2}\right)^{1 / 2} d \phi_{n} R .
\end{aligned}
$$

The proof is complete.

4.7 REMARK. The preceding corollary affords a new method for the evaluation of $\beta(n, m)$ which differs from the method in $[\mathrm{F} 1,5]$. Roughly speaking, by means of a "distribution measure," we can express

$$
\int_{G_{n}}\left(\sum_{j=1}^{m+1}\left|R_{j}^{n}\right|^{2}\right)^{1 / 2} d \phi_{n} R
$$

as the integral of a continuous function over the $(n-1)$-dimensional surface of the unit sphere in $E^{n}$. Thus $\beta(n, m+1)(\beta(n-1, m))^{-1}$ can be effectively evaluated. Furthermore, it is quite easy to evaluate $\beta(n, 1)$ for all $n$. From this information, $\beta(n, m)$ can be immediately calculated. The details follow.

Let

$$
S=E^{n} \cap \underset{x}{E}[|x|=1] .
$$

Statement 1. For $n \geqq m>1$,

$$
\frac{\beta(n, m)}{\beta(n-1, m-1)}=\frac{\Gamma\left(\frac{n}{2}\right) \cdot \Gamma\left(\frac{m+1}{2}\right)}{\Gamma\left(\frac{n+1}{2}\right) \cdot \Gamma\left(\frac{m}{2}\right)} .
$$

Proof. We let $\psi$ be the measure over $S$ defined by

$$
\psi(X)=\phi_{n}\left(G_{n} \cap \underset{R}{E}\left[R^{n} \in X\right]\right) \quad \text { for } X \subset S .
$$


252

GERALD FREILICH

[September

Then by 4.6 and 4.1 ,

$$
\begin{aligned}
\frac{\beta(n, m)}{\beta(n-1, m-1)} & =\int_{G_{n}}\left(\sum_{j=1}^{m}\left|R_{j}^{n}\right|^{2}\right)^{1 / 2} d \phi_{n} R \\
& =\left(\mathcal{F C}_{n}^{n-1}(S)\right)^{-1} \cdot \int_{S}\left(\sum_{j=1}^{m}\left|x_{j}\right|^{2}\right)^{1 / 2} d \mathcal{S C}_{n}^{n-1} x .
\end{aligned}
$$

Using generalized spherical coordinates $\left(\rho, \theta_{1}, \cdots, \theta_{n-1}\right)$ of a point $x \in E^{n}$, given by the relations

$$
\begin{aligned}
x_{n} & =\rho \cos \theta_{n-1}, \\
x_{n-1} & =\rho \sin \theta_{n-1} \cos \theta_{n-2}, \\
x_{n-2} & =\rho \sin \theta_{n-1} \sin \theta_{n-2} \cos \theta_{n-3}, \\
\cdot . & \cdot \ldots \cdot \ldots \cdot \ldots \cdot \ldots \\
x_{2} & =\rho \cdot \prod_{j=2}^{n-1} \sin \theta_{j} \cdot \cos \theta_{1}, \\
x_{1} & =\rho \cdot \prod_{j=1}^{n-1} \sin \theta_{j},
\end{aligned}
$$

we have

$$
\begin{aligned}
& \left(\operatorname{Fe}_{n}^{n-1}(S)\right)^{-1} \cdot \int_{S}\left(\sum_{j=1}^{m}\left|x_{j}\right|^{2}\right)^{1 / 2} d \operatorname{Fe}_{n}^{n-1} x \\
& =\frac{\int_{0}^{\pi} \int_{0}^{\pi} \cdots \int_{0}^{\pi} \int_{0}^{2 \pi}\left(\prod_{j=1}^{n-1} \sin ^{2} \theta_{j}+\sum_{j=2}^{m}\left(\prod_{i=j}^{n-1} \sin ^{2} \theta_{i}\right) \cos ^{2} \theta_{j-1}\right)^{1 / 2}}{\left(\prod_{j=1}^{n-2} \sin ^{j} \theta_{j+1}\right) d \theta_{1} d \theta_{2} \cdots d \theta_{n-2} d \theta_{n-1}} \\
& =\frac{\left(\prod_{j=m}^{n-1} \int_{0}^{\pi} \sin ^{j} \theta_{j} d \theta_{j}\right) \cdot\left(\prod_{j=2}^{m-1} \int_{0}^{\pi} \sin ^{j-1} \theta_{j} d \theta_{j}\right) \cdot 2 \pi}{\left(\prod_{j=1}^{n-2} \sin ^{j} \theta_{j+1}\right) d \theta_{1} d \theta_{2} \cdots d \theta_{n-2} d \theta_{n-1}} \\
& =\frac{\left.\int_{0}^{\pi-1} \int_{0}^{\pi} \sin ^{j-1} \theta_{j} d \theta_{j}\right) \cdot 2 \pi}{\int_{0}^{\pi-1} x d x} \sin ^{m-1} x d x
\end{aligned}
$$




$$
\begin{aligned}
& =\frac{\Gamma\left(\frac{n}{2}\right) \Gamma\left(\frac{1}{2}\right) \Gamma\left(\frac{n+1}{2}\right)^{-1}}{\Gamma\left(\frac{m}{2}\right) \Gamma\left(\frac{1}{2}\right) \Gamma\left(\frac{m+1}{2}\right)^{-1}} \\
& =\frac{\Gamma\left(\frac{n}{2}\right) \Gamma\left(\frac{m+1}{2}\right)}{\Gamma\left(\frac{m}{2}\right) \Gamma\left(\frac{n+1}{2}\right)} .
\end{aligned}
$$

This completes the proof of Statement 1.

Statement 2. $\beta(n, 1)=\Gamma(n / 2) \cdot \Gamma((n+1) / 2)^{-1} \cdot \Gamma(1 / 2)^{-1}$.

Proof. By definition 2.6,

$$
\beta(n, 1)=\int_{G_{n}} \Delta\left(\left.R\right|_{1} ^{1}\right) d \phi_{n} R=\int_{G_{n}}\left|R_{1}^{1}\right| d \phi_{n} R .
$$

By 4.1,

$$
\begin{aligned}
\int_{G_{n}}\left|R_{1}^{1}\right| d \phi_{n} R & =\left(\mathcal{F C}_{n}^{n-1}(S)\right)^{-1} \cdot \int_{S}\left|x_{1}\right| d \mathcal{H}_{n}^{n-1} x \\
& =\frac{\int_{0}^{\pi} \int_{0}^{\pi} \cdots \int_{0}^{\pi} \int_{0}^{2 \pi} \sin ^{n-1} \theta_{n-1} \sin ^{n-2} \theta_{n-2} \cdots \sin ^{2} \theta_{2}}{\int_{0}^{\pi} \int_{0}^{\pi} \cdots \int_{0}^{\pi} \int_{0}^{2 \pi} \sin ^{n-2} \theta_{n-1} \sin ^{n-3} \theta_{n-2} \cdots \sin \theta_{2}} \cdot d \theta_{1} d \theta_{2} \cdots d \theta_{n-2} d \theta_{n-1} \\
& =\frac{2 \cdot \prod_{j=1}^{n-1}\left(\int_{0}^{\pi} \sin ^{j} x d x\right)}{2 \pi \cdot \prod_{j=1}^{n-2}\left(\int_{0}^{\pi} \sin ^{j} x d x\right)} \\
& =\pi^{-1} \cdot \int_{0}^{\pi} \sin ^{n-1} x d x \\
& =\pi^{-1} \cdot \Gamma(n / 2) \cdot \Gamma(1 / 2) \cdot \Gamma((n+1) / 2)^{-1} \\
& =\Gamma(n / 2) \cdot \Gamma((n+1) / 2)^{-1} \cdot \Gamma(1 / 2)^{-1},
\end{aligned}
$$

hence

$$
\beta(n, 1)=\Gamma(n / 2) \cdot \Gamma((n+1) / 2)^{-1} \cdot \Gamma(1 / 2)^{-1}
$$


Statement 3.

$$
\beta(n, m)=\frac{\Gamma\left(\frac{m+1}{2}\right) \cdot \Gamma\left(\frac{n-m+1}{2}\right)}{\Gamma\left(\frac{n+1}{2}\right) \cdot \Gamma\left(\frac{1}{2}\right)} .
$$

Proof.

$$
\begin{aligned}
\beta(n, m)= & \frac{\beta(n, m)}{\beta(n-1, m-1)} \cdot \frac{\beta(n-1, m-1)}{\beta(n-2, m-2)} \ldots \\
& \cdot \frac{\beta(n-m+2,2)}{\beta(n-m+1,1)} \cdot \beta(n-m+1,1) \\
= & \frac{\Gamma\left(\frac{m+1}{2}\right) \Gamma\left(\frac{n}{2}\right)}{\Gamma\left(\frac{n+1}{2}\right) \Gamma\left(\frac{m}{2}\right)} \cdot \frac{\Gamma\left(\frac{m}{2}\right) \Gamma\left(\frac{n-1}{2}\right)}{\Gamma\left(\frac{n}{2}\right) \Gamma\left(\frac{m-1}{2}\right)} \ldots \\
& \cdot \frac{\Gamma\left(\frac{3}{2}\right) \Gamma\left(\frac{n-m+2}{2}\right)}{\Gamma\left(\frac{n-m+3}{2}\right) \Gamma(1)} \cdot \frac{\Gamma\left(\frac{n-m+1}{2}\right)}{\Gamma\left(\frac{1}{2}\right) \Gamma\left(\frac{n-m+2}{2}\right)} \\
= & \frac{\Gamma\left(\frac{m+1}{2}\right) \Gamma\left(\frac{n-m+1}{2}\right)}{\Gamma\left(\frac{n+1}{2}\right) \Gamma\left(\frac{1}{2}\right)} \cdot
\end{aligned}
$$

This completes the proof.

5. The integralgeometric (Favard) measure of product sets. In this section we consider the case in which $\beta=k$.

5.1 LемMA. If

$1 \leqq k \leqq n, 0 \leqq m \leqq n-k ; m, n$, and $k$ are integers,

$A=E^{n} \cap \underset{x}{E}\left[x_{n-k+1}=\cdots=x_{n}=0\right]$,

$B=E^{n} \cap \underset{x}{E}\left[x_{1}=\cdots=x_{n-k}=0\right]$,

$S \subset A, S$ is analytic, $\mathcal{F}_{n}^{m}(S)<\infty$,

$T=B \cap \underset{x}{E}\left[x_{n-k+i} \in J_{i}\right.$ for $\left.i=1, \cdots, k\right]$,

where $J_{i}$ is an analytic subset of $E^{1}$ of finite $\mathcal{L}_{1}$ measure, for $i=1, \cdots, k$, then 


$$
\mathcal{F}_{n}^{m+k}(S+T)=\mathcal{F}_{n}^{m}(S) \cdot \mathcal{F}_{n}^{k}(T) .
$$

Proof. Note first that (5) implies that $T \subset B$ is analytic.

The lemma will be proved by induction on $k$.

For $k=1$ and for arbitrary $n, m$ with $0 \leqq m \leqq n-1$, then 4.5 implies that $\mathcal{F}_{n}^{m+1}(S+T)=\mathcal{F}_{n}^{m}(S) \cdot \mathcal{F}_{n}^{1}(T)<\infty$, since $\mathcal{F}_{n}^{1}(T)=\mathcal{L}_{1}\left(J_{1}\right)<\infty$.

Suppose now that the lemma holds for all $k$ such that $1 \leqq k \leqq q$ and for all $n, m$, such that $0 \leqq m \leqq n-k$. Let $k=q+1$ and let $m, n$ be such integers that $0 \leqq m \leqq n-k$. By the inductive hypothesis, we have

$$
\mathcal{F}_{n-1}^{m+q}\left(p_{n}^{n-1 *}(S)+p_{n}^{n-1 *}(T)\right)=\mathcal{F}_{n-1}^{m}\left(p_{n}^{n-1 *}(S)\right) \cdot \mathcal{F}_{n-1}^{q}\left(p_{n}^{n-1 *}(T)\right)<\infty,
$$

since $\mathcal{F}_{n-1}^{m}\left(p_{n}^{n-1 *}(S)\right)=\mathcal{F}_{n}^{m}(S)<\infty$. Let

$$
\begin{aligned}
& X=\left(\eta_{n}^{n-1}: p_{n}^{n-1}\right)^{*}(S+T), \\
& Y=E^{n} \cap \underset{x}{E}\left[x_{1}=\cdots=x_{n-1}=0, x_{n} \in J_{k}\right] .
\end{aligned}
$$

Now

$$
\mathcal{F}_{n}^{m+q}(X)=\mathcal{F}_{n-1}^{m+q}\left(p_{n}^{n-1 *}(S)+p_{n}^{n-1 *}(T)\right),
$$

and since we are essentially dealing with Lebesgue measures,

$$
\mathcal{F}_{n}^{1}(Y) \cdot \mathcal{F}_{n-1}^{q}\left(p_{n}^{n-1 *}(T)\right)=\mathcal{F}_{n}^{k}(T) .
$$

Also 4.5 implies that

$$
\mathcal{F}_{n}^{m+k}(X+Y)=\mathcal{F}_{n}^{m+q}(X) \cdot \mathcal{F}_{n}^{1}(Y)<\infty,
$$

therefore

$$
\begin{aligned}
\mathcal{F}_{n}^{m+k}(X+Y) & =\mathcal{F}_{n}^{m}(S) \cdot \mathcal{F}_{n-1}^{q}\left(p_{n}^{n-1 *}(T)\right) \cdot \mathcal{F}_{n}^{1}(Y) \\
& =\mathcal{F}_{n}^{m}(S) \cdot \mathcal{F}_{n}^{k}(T) .
\end{aligned}
$$

But $X+Y=S+T$. Hence

$$
\mathcal{F}_{n}^{m+k}(S+T)=\mathcal{F}_{n}^{m}(S) \cdot \mathcal{F}_{n}^{k}(T)<\infty,
$$

which completes the proof.

5.2 LemMa. If conditions (1), (2), (3), (4) of 5.1 are satisfied and

$$
T \subset B, \mathcal{f}_{n}^{k}(T)<\infty,\left(E^{k} \cap \underset{x}{E}\left[\left(0, \cdots, 0, x_{1}, \cdots, x_{k}\right) \in T\right]\right)
$$

is an open subset of $E^{k}$, then

$$
\mathcal{F}_{n}^{m+k}(S+T)=\mathcal{F}_{n}^{m}(S) \cdot \mathcal{F}_{n}^{k}(T) .
$$


Proof. Let

$$
T^{\prime}=E^{k} \cap \underset{x}{E}\left[\left(0, \cdots, 0, x_{1}, \cdots, x_{k}\right) \in T\right]
$$

Then by standard methods, we can obtain a decomposition of $T^{\prime}$ in to a countable number of parallelotopes (not necessarily open or closed) with "sides" parallel to the coordinate axes. More precisely, we may express

$$
T^{\prime}=\bigcup_{i=1}^{\infty} T_{i}^{\prime}
$$

such that

$$
\begin{array}{ll}
T_{i}^{\prime} \cap T_{j}^{\prime}=\varnothing & \text { for } i \neq j \\
T_{i}^{\prime}=E^{k} \cap \underset{x}{E}\left[x_{i} \in J_{i}^{i} \text { for } j=1, \cdots, k\right], &
\end{array}
$$

where $J_{j}^{4}$ is a bounded interval of $E^{1}$ for $i=1,2,3, \cdots ; j=1, \cdots, k$. Let

$$
T_{i}=E^{n} \cap \underset{x}{E}\left[x_{1}=\cdots=x_{n-k}=0 \text { and }\left(x_{n-k+1}, \cdots, x_{n}\right) \in T_{i}^{\prime}\right] \text {, }
$$

for $i=1,2,3, \cdots$. Then by $5.1, \mathcal{F}_{m}^{m+k}\left(S+T_{i}\right)=\mathcal{F}_{n}^{m}(S) \cdot \mathcal{F}_{n}^{k}\left(T_{i}\right)$, for $i=1$, 2, 3, ... Moreover,

$$
\begin{array}{rlrl}
T & =\bigcup_{i=1}^{\infty} T_{i}, & \\
T_{i} \cap T_{j} & =\varnothing & & \\
S+T & =\bigcup_{i=1}^{\infty}\left(S+T_{i}\right), & \\
\left(S+T_{i}\right) \cap\left(S+T_{j}\right) & =\varnothing & \text { for } i \neq j,
\end{array}
$$

and therefore

$$
\begin{aligned}
\mathcal{F}_{n}^{m+k}(S+T) & =\sum_{i=1}^{\infty} \mathcal{F}_{n}^{m+k}\left(S+T_{i}\right) \\
& =\sum_{i=1}^{\infty} \mathcal{F}_{n}^{m}(S) \cdot \mathcal{F}_{n}^{k}\left(T_{i}\right) \\
& =\mathcal{F}_{n}^{m}(S) \cdot \sum_{i=1}^{\infty} \mathcal{F}_{n}^{k}\left(T_{i}\right) \\
& =\mathcal{F}_{n}^{m}(S) \cdot \mathcal{F}_{n}^{k}(T) .
\end{aligned}
$$

The proof is complete. 
5.3 Theorem. If conditions (1), (2), (3), (4) of 5.1 are satisfied and $\left(5^{\prime \prime}\right)$ $T \subset B, T$ is analytic, $\mathcal{F}_{n}^{k}(T)<\infty$, then $\mathcal{F}_{n}^{m+k}(S+T)=\mathcal{F}_{n}^{m}(S) \cdot \mathcal{F}_{n}^{k}(T)$.

Proof. Let

$$
T^{\prime}=E^{k} \cap \underset{x}{E}\left[\left(0, \cdots, 0, x_{1}, \cdots, x_{k}\right) \in T\right] .
$$

Then $T^{\prime}$ is analytic, hence $\mathcal{L}_{k}$ measurable, and

$$
\mathcal{L}_{k}\left(T^{\prime}\right)=\mathcal{F}_{n}^{k}(T)<\infty .
$$

Let $\epsilon>0$ and choose a set $W_{1}$, open in $E^{k}$, such that

$$
T^{\prime} \subset W_{1} \text { and } \mathcal{L}_{k}\left(W_{1}\right)<\mathcal{L}_{k}\left(T^{\prime}\right)+\epsilon .
$$

Now $\left(W_{1}-T^{\prime}\right)$ is $\mathcal{L}_{k}$ measurable and

$$
\mathcal{L}_{k}\left(W_{1}-T^{\prime}\right)=\mathcal{L}_{k}\left(W_{1}\right)-\mathcal{L}_{k}\left(T^{\prime}\right)<\epsilon .
$$

Hence we may choose $W_{2}$, open in $E^{k}$, such that

$$
\begin{gathered}
\left(W_{1}-T^{\prime}\right) \subset W_{2} \subset W_{1}, \\
\mathcal{L}_{k}\left(W_{2}\right)<\mathcal{L}_{k}\left(W_{1}-T^{\prime}\right)+\epsilon<2 \epsilon .
\end{gathered}
$$

For $i=1,2$, define

$$
W_{i}^{\prime}=E^{n} \cap \underset{x}{E}\left[x_{1}=\cdots=x_{n-k}=0 \text { and }\left(x_{n-k+1}, \cdots, x_{n}\right) \in W_{i}\right] .
$$

Then by 5.2 we have, for $i=1,2$,

$$
\mathcal{F}_{n}^{m+k}\left(S+W_{i}^{\prime}\right)=\mathcal{F}_{n}^{m}(S) \cdot \mathcal{F}_{n}^{k}\left(W_{i}^{\prime}\right)=\mathcal{F}_{n}^{m}(S) \cdot \mathcal{L}_{k}\left(W_{i}\right) .
$$

Since $S+\left(W_{1}^{\prime}-W_{2}^{\prime}\right) \subset(S+T) \subset\left(S+W_{1}^{\prime}\right)$, it follows that

$$
\mathcal{F}_{n}^{m+k}\left(S+\left(W_{1}^{\prime}-W_{2}^{\prime}\right)\right) \leqq \mathcal{F}_{n}^{m+k}(S+T) \leqq \mathcal{F}_{n}^{m+k}\left(S+W_{1}^{\prime}\right)
$$

Hence

$$
\begin{aligned}
\mathcal{F}_{n}^{m}(S) \cdot\left(\mathcal{L}_{k}\left(W_{1}\right)-2 \epsilon\right) & \leqq \mathcal{F}_{n}^{m}(S) \cdot\left(\mathcal{L}_{k}\left(W_{1}\right)-\mathcal{L}_{k}\left(W_{2}\right)\right) \\
& =\mathcal{F}_{n}^{m+k}\left(S+W_{1}^{\prime}\right)-\mathcal{F}_{n}^{m+k}\left(S+W_{2}^{\prime}\right) \\
& =\mathcal{F}_{n}^{m+k}\left(S+\left(W_{1}^{\prime}-W_{2}^{\prime}\right)\right) \\
& \leqq \mathcal{F}_{n}^{m+k}(S+T) \\
& \leqq \mathcal{F}_{n}^{m+k}\left(S+W_{1}^{\prime}\right) \\
& =\mathcal{F}_{n}^{m}(S) \cdot \mathcal{L}_{k}\left(W_{1}\right)
\end{aligned}
$$


By the arbitrary nature of $\epsilon$ and because

$$
\mathcal{L}_{k}\left(W_{1}\right)-2 \epsilon \leqq \mathcal{L}_{k}\left(W_{1}-W_{2}\right) \leqq \mathcal{L}_{k}\left(T^{\prime}\right)=\mathcal{F}_{n}^{k}(T)=\mathcal{L}_{k}\left(T^{\prime}\right) \leqq \mathcal{L}_{k}\left(W_{1}\right),
$$

we conclude that

$$
\mathcal{F}_{n}^{m+k}(S+T)=\mathcal{F}_{n}^{m}(S) \cdot \mathcal{F}_{n}^{k}(T) .
$$

5.4 REMARK. Though the methods used in the proof of 4.5 can be generalized to prove 5.3 , the above proof is simpler and more elegant.

5.5 THEOREM. If

(1) $A$ is a subspace of $E^{n}$ of dimension $(n-k), 1 \leqq k<n$,

(2) $B$ is the subspace of $E^{n}$ of dimension $k$, orthogonal to $A$,

(3) $0 \leqq m \leqq n-k$,

(4) $S \subset A, S$ is countably $\mathcal{F}_{n}^{m}$ measurable,

then

(5) $T \subset B, T$ is countably $\mathcal{F}_{n}^{k}$ measurable,

$$
\mathcal{F}_{n}^{m+k}(S+T)=\mathcal{F}_{n}^{m}(S) \cdot \mathcal{F}_{n}^{k}(T) .
$$

Proof. By 2.10, we may just as well assume that

$$
\begin{aligned}
A & =E^{n} \cap \underset{x}{E}\left[x_{n-k+1}=\cdots=x_{n}=0\right], \\
B & =E^{n} \cap \underset{x}{E}\left[x_{1}=\cdots=x_{n-k}=0\right] .
\end{aligned}
$$

Assume further that $\mathcal{F}_{n}^{m}(S)<\infty, \mathcal{F}_{n}^{k}(T)<\infty$. Then since $S$ and $T$ are respectively $\mathcal{F}_{n}^{m}$ and $\mathcal{F}_{n}^{k}$ measurable sets, we can find analytic sets $U_{1}$ and $V_{1}$ such that

$$
\begin{array}{ll}
S \subset U_{1} \subset A, & T \subset V_{1} \subset B, \\
\mathcal{F}_{n}^{m}(S)=\mathcal{F}_{n}^{m}\left(U_{1}\right), & \mathcal{F}_{n}^{k}(T)=\mathcal{F}_{n}^{k}\left(V_{1}\right) .
\end{array}
$$

Hence

$$
\mathcal{F}_{n}^{m}\left(U_{1}-S\right)=0, \quad \mathcal{F}_{n}^{k}\left(V_{1}-T\right)=0 .
$$

Choose analytic sets $U_{2}$ and $V_{2}$ such that

$$
\begin{array}{ll}
\left(U_{1}-S\right) \subset U_{2} \subset A, & \left(V_{1}-T\right) \subset V_{2} \subset B, \\
\mathcal{f}_{n}^{m}\left(U_{2}\right)=0, & \mathcal{f}_{n}^{k}\left(V_{2}\right)=0 .
\end{array}
$$

Then

$$
\begin{aligned}
& U_{1}-U_{2} \subset S, \quad V_{1}-V_{2} \subset T, \\
& \mathcal{F}_{n}^{m}\left(U_{1}-U_{2}\right)=\mathcal{F}_{n}^{m}\left(U_{1}\right)=\mathcal{F}_{n}^{m}(S), \\
& \mathcal{F}_{n}^{k}\left(V_{1}-V_{2}\right)=\mathcal{F}_{n}^{k}\left(V_{1}\right)=\mathcal{F}_{n}^{k}(T) .
\end{aligned}
$$


By 5.3, we have

$$
\begin{aligned}
\mathcal{F}_{n}^{m+k}\left(U_{1}+V_{1}\right)=\mathcal{F}_{n}^{m}\left(U_{1}\right) \cdot \mathcal{F}_{n}^{k}\left(V_{1}\right) & =\mathcal{F}_{n}^{m}(S) \cdot \mathcal{F}_{n}^{k}(T), \\
\mathcal{F}_{n}^{m+k}\left(\left(U_{1}-U_{2}\right)+\left(V_{1}-V_{2}\right)\right) & =\mathcal{F}_{n}^{m}\left(U_{1}-U_{2}\right) \cdot \mathcal{F}_{n}^{k}\left(V_{1}-V_{2}\right) \\
& =\mathcal{F}_{n}^{m}(S) \cdot \mathcal{F}_{n}^{k}(T) .
\end{aligned}
$$

Finally,

$$
\begin{aligned}
\mathcal{F}_{n}^{m}(S) \cdot \mathcal{F}_{n}^{k}(T) & =\mathcal{F}_{n}^{m+k}\left(\left(U_{1}-U_{2}\right)+\left(V_{1}-V_{2}\right)\right) \\
& \leqq \mathcal{F}_{n}^{m+k}(S+T) \\
& \leqq \mathcal{F}_{n}^{m+k}\left(U_{1}+V_{1}\right) \\
& =\mathcal{F}_{n}^{m}(S) \cdot \mathcal{F}_{n}^{k}(T),
\end{aligned}
$$

therefore

$$
\mathcal{F}_{n}^{m+k}(S+T)=\mathcal{F}_{n}^{m}(S) \cdot \mathcal{F}_{n}^{k}(T) .
$$

If now $S$ is countably $\mathcal{F}_{n}^{m}$ measurable, then we may express

$$
S=\bigcup_{i=1}^{\infty} S_{i}, \quad S_{i} \cap S_{j}=\varnothing \quad \text { for } i \neq j,
$$

$S_{i}$ is $\mathcal{F}_{n}^{m}$ measurable and $\mathcal{F}_{n}^{m}\left(S_{i}\right)<\infty$ for $i=1,2, \ldots$. Similarly if $T$ is countably $\mathcal{F}_{n}^{k}$ measurable, then we may write

$$
T=\bigcup_{i=1}^{\infty} T_{i}, \quad T_{i} \cap T_{i}=\varnothing \quad \text { for } i \neq j,
$$

$T_{i}$ is $\mathcal{F}_{n}^{k}$ measurable and $\mathcal{F}_{n}^{k}\left(T_{i}\right)<\infty$ for $i=1,2, \cdots$. Then

$$
\begin{aligned}
\mathcal{F}_{n}^{m+k}(S+T) & =\mathcal{F}_{n}^{m+k}\left(\bigcup_{i=1}^{\infty} S_{i}+\bigcup_{i=1}^{\infty} T_{i}\right) \\
& =\mathcal{F}_{n}^{m+k}\left(\bigcup_{i=1}^{\infty} \bigcup_{j=1}^{\infty}\left(S_{i}+T_{j}\right)\right) \\
& =\sum_{i=1}^{\infty} \sum_{j=1}^{\infty} \mathcal{F}_{n}^{m+k}\left(S_{i}+T_{j}\right) \\
& =\sum_{i=1}^{\infty} \sum_{j=1}^{\infty} \mathcal{F}_{n}^{m}\left(S_{i}\right) \cdot \mathcal{F}_{n}^{k}\left(T_{j}\right) \\
& =\left(\sum_{i=1}^{\infty} \mathcal{F}_{n}^{m}\left(S_{i}\right)\right) \cdot \mathcal{F}_{n}^{k}(T) \\
& =\mathcal{F}_{n}^{m}(S) \cdot \mathcal{F}_{n}^{k}(T) .
\end{aligned}
$$


The proof is complete.

5.6 REMARK. If in the hypothesis of 5.5 we do not require $S$ and $T$ to be $\mathcal{F}_{n}^{m}$ and $\mathcal{F}_{n}^{k}$ measurable respectively, then it is obvious that

$$
\mathcal{F}_{n}^{m+k}(S+T) \leqq \mathcal{F}_{n}^{m}(S) \cdot \mathcal{F}_{n}^{k}(T) .
$$

5.7 THEOREM. If

(1) $A$ is a subspace of $E^{n}$ of dimension $\alpha$,

(2) $B$ is the subspace of $E^{n}$ of dimension $\beta=n-\alpha$, orthogonal to $A$,

(3) $0 \leqq m \leqq \alpha, 0 \leqq k \leqq \beta$,

(4) $f$ is a Lipschitzian function, $\operatorname{dmn} f=X$, where $X$ is an $\mathcal{L}_{m}$ measurable subset of $E^{m}, f^{*}(X)=S \subset A$,

(5) $g$ is a Lipschitzian function, dmn $g=Y$, where $Y$ is an $\mathcal{L}_{k}$ measurable subset of $E^{k}, g^{*}(Y)=T \subset B$,

then

$$
\mathcal{F}_{n}^{m+k}(S+T)=\mathcal{F}_{n}^{m}(S) \cdot \mathcal{F}_{n}^{k}(T) .
$$

Proof. Without loss of generality, we may assume that

$$
\begin{aligned}
& A=E^{n} \cap \underset{x}{E}\left[x_{\alpha+1}=\cdots=x_{n}=0\right], \\
& B=E^{n} \cap \underset{x}{E}\left[x_{1}=\cdots=x_{\alpha}=0\right] .
\end{aligned}
$$

By [MF], we may further assume that both $f$ and $g$ are univalent.

Now for all functions $u, v$, such that dmn $u \subset E^{m}$, dmn $v \subset E^{k}$, rng $u \subset E^{n}$, rng $v \subset E^{n}$, we define the function $(u \otimes v)$ on (dmn $\left.u \times \operatorname{dmn} v\right) \subset E^{m+k}$ (we shall make no distinction between $E^{m} \times E^{k}$ and $E^{m+k}$ ) to $E^{n}$ by the formula

$$
(u \otimes v)(x)=u\left(x_{1}, \cdots, x_{m}\right)+v\left(x_{m+1}, \cdots, x_{m+k}\right)
$$

for $x=\left(x_{1}, \cdots, x_{m+k}\right) \in \mathrm{dmn}(u \otimes v)$. Then

$(f \otimes g)$ is univalent and Lipschitzian,

$(f \otimes g) *(X \times Y)=(S+T)$.

Letting $D h(x)$ denote the (total) differential of $h$ at $x$, then by straightforward methods it is seen that for $\mathcal{L}_{m+k}$ almost all $z \in(X \times Y)$,

$$
D(f \otimes g)(z)=D f\left(z_{1}, \cdots, z_{m}\right) \otimes D g\left(z_{m+1}, \cdots, z_{m+k}\right),
$$

and therefore $J(f \otimes g)(z)=J f\left(z_{1}, \cdots, z_{m}\right) \cdot J g\left(z_{m+1}, \cdots, z_{m+k}\right)$. Hence by $[\mathrm{F} 2,5.13]$,

$$
\begin{aligned}
\mathcal{F}_{n}^{m+k}(S+T) & =\int_{E^{n}} N[(f \otimes g),(X \times Y), z] d \mathcal{F}_{n}^{m+k} z \\
& =\int_{X \times \mathbf{Y}} J(f \otimes g)(z) d \mathcal{L}_{m+k} z
\end{aligned}
$$




$$
\begin{aligned}
& =\int_{X} J f(x) d \mathcal{L}_{m} x \cdot \int_{Y} J g(y) d \mathcal{C}_{k} y \\
& =\int_{E^{n}} N[f, X, x] d \mathcal{F}_{n}^{m} x \cdot \int_{E^{n}} N[g, Y, y] d \mathcal{F}_{n}^{k} y \\
& =\mathcal{F}_{n}^{m}(S) \cdot \mathcal{F}_{n}^{k}(T) .
\end{aligned}
$$

The proof is complete.

\subsection{THEOREM. If}

(1) $A$ is a subspace of $E^{n}$ of dimension $\alpha$,

(2) $B$ is the subspace of $E^{n}$ of dimension $\beta=n-\alpha$, orthogonal to $A$,

(3) $0 \leqq m \leqq \alpha, 0 \leqq k \leqq \beta$,

(4) $S \subset A$ is a countable union of $m$ rectifiable, $\mathcal{F}_{n}^{m}$ measurable sets of finite $\mathcal{F}_{n}^{m}$ measure,

(5) $T \subset B$ is a countable union of $k$ rectifiable, $\mathcal{F}_{n}^{k}$ measurable sets of finite $\mathcal{F}_{n}^{\mathrm{k}}$ measure,

then

$$
\mathcal{F}_{n}^{m+k}(S+T)=\mathcal{F}_{n}^{m}(S) \cdot \mathcal{F}_{n}^{k}(T) .
$$

Proof. We may just as well assume that $S$ is $m$ rectifiable, $\mathcal{F}_{n}^{m}(S)<\infty$, $T$ is $k$ rectifiable, $\mathcal{F}_{n}^{k}\left(T^{\prime}\right)<\infty$.

Since the closure of a rectifiable set is rectifiable, we may choose analytic $m$ rectifiable sets $S^{\prime}$ and $S^{\prime \prime}$ for which

$$
S^{\prime \prime} \subset S \subset S^{\prime} \subset A, \quad \mathcal{f}_{n}^{m}\left(S^{\prime \prime}\right)=\mathcal{F}_{n}^{m}(S)=\mathcal{F}_{n}^{m}\left(S^{\prime}\right) \text {. }
$$

By 2.11, choose a Lipschitzian function $f$ such that $\operatorname{dmn} f=E^{m}, f^{*}\left(E^{m}\right)$ $\supset S^{\prime} \supset S^{\prime \prime}$. By the analyticity of $S^{\prime}$ and $S^{\prime \prime}$ and the continuity of $f$,

$$
\begin{aligned}
X^{\prime} & =\left(E^{m} \cap \underset{x}{E}\left[f(x) \in S^{\prime}\right]\right) \text { is an analytic set, } \\
X^{\prime \prime} & =\left(E^{m} \cap \underset{x}{E}\left[f(x) \in S^{\prime \prime}\right]\right) \text { is an analytic set. }
\end{aligned}
$$

Let $f_{1}=\left(f \mid X^{\prime}\right), f_{2}=\left(f \mid X^{\prime \prime}\right)$,

Similarly choose Lipschitzian functions $g_{1}, g_{2}$, with $\mathcal{L}_{k}$ measurable domains and sets $T^{\prime}, T^{\prime \prime}$, such that

$$
\operatorname{rng} g_{2}=T^{\prime \prime} \subset T \subset T^{\prime}=\operatorname{rng} g_{1} \subset B, \quad \mathcal{f}_{n}^{k}\left(T^{\prime \prime}\right)=\mathcal{f}_{n}^{k}(T)=\mathcal{f}_{n}^{k}\left(T^{\prime}\right) .
$$

Then by 5.7,

$$
\begin{aligned}
\mathcal{F}_{n}^{m}(S) \cdot \mathcal{F}_{n}^{k}(T) & =\mathcal{F}_{n}^{m}\left(S^{\prime \prime}\right) \cdot \mathcal{F}_{n}^{k}\left(T^{\prime \prime}\right)=\mathcal{F}_{n}^{m+k}\left(S^{\prime \prime}+T^{\prime \prime}\right) \\
& \leqq \mathcal{F}_{n}^{m+k}(S+T) \leqq \mathcal{F}_{n}^{m+k}\left(S^{\prime}+T^{\prime}\right)=\mathcal{F}_{n}^{m}\left(S^{\prime}\right) \cdot \mathcal{F}_{n}^{k}\left(T^{\prime}\right) \\
& =\mathcal{F}_{n}^{m}(S) \cdot \mathcal{F}_{n}^{k}(T) .
\end{aligned}
$$


The proof is complete.

6. Hausdorff measure of product sets. In this section we construct a subset $A$ of $E^{2}$ such that the unit cylinder $B$ with base $A$ has larger 2-dimensional Hausdorff measure than the 1-dimensional Hausdorff measure of $A$. In doing this we use and extend some of the ideas of Besicovitch and Moran, who gave a rough outline of such a construction (see $[B M]$ ).

6.1 Definition. If $C$ is a subset of $E^{3}$, we shall say that

$D$ is complete with respect to $C$,

if and only if $D$ is such a bounded subset of $C$ that

$$
(p \in C-D) \rightarrow(\operatorname{diam}(D \cup\{p\})>\operatorname{diam} D) .
$$

Unless otherwise stated, we shall mean in this paper by a complete set, a set which is complete with respect to $E^{3}$.

We shall make use of the fact that a set is complete (in $E^{3}$ ) if and only if it has constant width.

6.2 Remark. For the properties of convex sets and, in particular, complete sets, which we shall use, see $[\mathrm{BF}]$.

6.3 Theorem. Let $a>0$,

$$
C=E^{3} \subset \underset{z}{E}\left[z_{1}^{2}+z_{2}^{2} \leqq a^{2} / 4\right],
$$

$\infty>d>0$. Then of all subsets of $C$ with diameter less than or equal to $d$, the set

$$
(C \cap \underset{z}{E}[|z| \leqq d / 2])
$$

has the largest volume.

Proof. Let $D \subset C$ be such that diam $D=\delta \leqq d$. Choose a convex set $D_{1} \subset C$ such that $D \subset D_{1}$ and diam $D_{1}=\delta$.

Let now $H_{1}$ be the supporting function of $D_{1}$ (see [BF, p. 23]). Then by the "central-symmetrization" of [BF, p. 73], we define the function $\mathrm{H}_{2}$ on $E^{3}$ by $H_{2}(u)=(1 / 2)\left(H_{1}(u)+H_{1}(-u)\right)$ for $u \in E^{3} . H_{2}$ is the supporting function of a convex set, call it $D_{2}$, with $\theta^{3}$ as center and with the properties that diam $D_{2}=\operatorname{diam} D_{1}=\delta, \mathcal{L}_{3}\left(D_{2}\right) \geqq \mathcal{L}_{3}\left(D_{1}\right)$. Moreover if $u \in E^{3},|u|=1, u_{3}=0$, then $\left|H_{1}(u)\right| \leqq a / 2$, since $D_{1} \subset C$. Hence $\left|H_{2}(u)\right| \leqq a / 2$, which implies that $D_{2} \subset C$.

Finally we shall show that

$$
D_{2} \subset(C \cap \underset{z}{E}[|z| \leqq d / 2]) .
$$

Let $z \in D_{2}$. Since $D_{2}$ has $\theta^{3}$ as center, $-z \in D_{2}$. Hence

$$
2 \cdot|z|=|z-(-z)| \leqq \operatorname{diam} D_{2}=\delta \leqq d, \quad|z| \leqq d / 2,
$$




$$
z \in C \cap \underset{z}{E}[|z| \leqq d / 2]
$$

Thus

$$
\mathcal{L}_{3}(C \cap \underset{z}{E}[|z| \leqq d / 2]) \geqq \mathcal{L}_{3}\left(D_{2}\right) \geqq \mathcal{L}_{3}(D),
$$

and the proof is complete.

6.4 REMARK. The isodiametric inequality proved in 6.3 can obviously be generalized to any cylinder symmetric with respect to its axis. However we shall use only the result stated in 6.3.

6.5 Definition. If $c$ is a bounded subset of $E^{3}$, let $g_{c}$ be the function on $E^{2}$ to $E^{1}$ such that for $x \in E^{2}$,

$$
g_{c}(x)=\mathcal{H}_{3}^{1}\left(c \cap \underset{z}{E}\left[z=\left(x_{1}, x_{2}, t\right), 0 \leqq t \leqq 1\right]\right) .
$$

6.6 TheOREM. If $c$ is a complete set of diameter $d$, and $\delta>0$, then

$\left(x \in E^{2}, y \in E^{2}\right.$ and $\left.|x-y| \leqq \delta\right) \rightarrow\left(\left|g_{c}(x)-g_{c}(y)\right| \leqq 2^{3 / 2} \cdot(\delta d)^{1 / 2}\right)$.

Proof. Let $c_{1}=p_{3}^{2 *}(c)$ and let $\beta$ be the boundary of $c_{1}$. The following fact is well known:

If $h$ is a convex function on the interval

$$
\left(E^{1} \cap \underset{x}{E}[a \leqq x \leqq b]\right)
$$

to $E_{1}$ and $\lambda>0$, then either

$$
\sup _{|x-a| \leqq \lambda}|h(x)-h(a)|=\sup _{|x-y| \leqq \lambda}|h(x)-h(y)|,
$$

or

$$
\sup _{|x-b| \leqq \lambda}|h(x)-h(b)|=\sup _{|x-y| \leqq \lambda}|h(x)-h(y)| .
$$

Suppose now that $x \in c_{1}, y \in c_{1}$, and $0<|x-y| \leqq \delta$. Choose $a \in c_{1}, b \in c_{1}$ so that

$$
\{a\} \cup\{b\}=\beta \cap \underset{z}{E}[z=y+t(x-y),-\infty<t<\infty]
$$

Since

$$
\left(g_{c} \mid \underset{z}{E}[z=a+t(b-a), 0 \leqq t \leqq 1]\right)
$$

is a convex function, we conclude that either

$$
\left|g_{c}(x)-g_{c}(y)\right| \leqq \sup _{|z-a| \leqq \delta}\left|g_{c}(z)-g_{c}(a)\right|
$$

or 


$$
\left|g_{c}(x)-g_{c}(y)\right| \leqq \sup _{|z-b| \leqq \delta}\left|g_{c}(z)-g_{c}(b)\right| .
$$

Hence it follows that

$$
\sup _{x \in c_{1}, y \in c_{1},|x-y| \leqq \delta}\left|g_{c}(x)-g_{c}(y)\right|=\sup _{x \in \beta, y \in c_{1},|x-y| \leqq \delta}\left|g_{c}(x)-g_{c}(y)\right| .
$$

Now let $x \in \beta, y \in c_{1}, 0<|x-y| \leqq \delta$. By [BF, p. 127] there is a unique number $t$ for which $\left(x_{1}, x_{2}, t\right) \in c$. Since $x$ is a boundary point of $c_{1}$, there is a supporting line $L$ of $c_{1}$ through $x$. Let $L^{\prime}$ be the line determined by $\left(x_{1}, x_{2}, 0\right)$ and $\left(x_{1}, x_{2}, 1\right)$. Then obviously $L$ and $L^{\prime}$ determine a vertical supporting plane to $c$ through $\left(x_{1}, x_{2}, t\right)$. Since $c$ is a set of constant width, we may choose $x^{\prime} \in c_{1}$ such that $\left|x^{\prime}-x\right|=d$, and such that $\left(x_{1}^{\prime}, x_{2}^{\prime}, t\right) \in c$. Therefore $c$ is contained in the sphere of radius $d$ and center $\left(x_{1}^{\prime}, x_{2}^{\prime}, t\right)$. The intersection of this sphere with the plane determined by $\left(x_{1}, x_{2}, 0\right),\left(y_{1}, y_{2}, 0\right)$, and $\left(y_{1}, y_{2}, 1\right)$ is then a disc of radius $r \leqq d$, whose circumference passes through $\left(x_{1}, x_{2}, t\right)$. Hence since the length of the vertical chord (of this disc) passing through $\left(y_{1}, y_{2}, t\right)$ is $2 \cdot\left(r^{2}-(r-|x-y|)^{2}\right)^{1 / 2}=2 \cdot\left(2 r \cdot|x-y|-|x-y|^{2}\right)^{1 / 2}$, we conclude that

$$
\left|g_{c}(x)-g_{c}(y)\right|=g_{c}(y) \leqq 2 \cdot\left(2 r \cdot|x-y|-|x-y|^{2}\right)^{1 / 2} \leqq 2 \cdot(2 d \delta)^{1 / 2} .
$$

Hence $\sup _{|x-y| \leqq \delta}\left|g_{c}(x)-g_{c}(y)\right| \leqq 2^{3 / 2} \cdot(\delta d)^{1 / 2}$. This completes the proof.

6.7 REMARK. If for each bounded subset $c$ of $E^{3}$ we let $g_{c}^{\prime}$ be the function on $E^{2}$ to $E^{1}$ such that

$$
g_{c}^{\prime}(x)=\mathfrak{H C}_{3}^{1}\left(c \cap \underset{z}{E}\left[z=\left(x_{1}, x_{2}, t\right),-\infty<t<\infty\right]\right),
$$

for $x \in E^{2}$, then 6.6 remains true with $g_{c}^{\prime}$ substituted for $g_{c}$.

6.8 Definition. Suppose $n$ is a positive integer, $B$ is a closed disc in $E^{2}$ of radius $a>0$ and with center $z \in E^{2}$. Let

$$
\begin{aligned}
& B_{0}=\left(E^{2} \cap \underset{x}{E}[|x| \leqq a]\right), \\
& C=\left(E^{2} \cap \underset{x}{E}\left[x_{1}=(j / n) a, x_{2}=(k / n) a, \text { where } j \text { and } k \text { are integers }\right]\right), \\
& N=\gamma\left(C \cap B_{0}\right),
\end{aligned}
$$

$f$ be the function on $E^{2}$ such that $f(x)=(1-1 / N) x$, for $x \in E^{2}$.

We shall say that

$A$ is the $n$-uniform spread of $B$,

if and only if

$$
A=\bigcup_{\nu \in f^{*}\left(C \cap \cap_{\left.B_{0}\right)}\right.}\left\{E^{2} \cap \underset{x}{E}[|x-y-z| \leqq a / N]\right\} .
$$

Since $N=\gamma(A)$ is obviously independent of the radius and center of $B$ and depends only on $n$, we shall call it $N(n)$. 
6.9 Remark. Let $B$ be a closed disc in $E^{2}$ of radius $a>0$, let $A_{n}$ denote the $n$-uniform spread of $B$ for $n=1,2,3, \cdots$, and let

$$
D_{n}=\inf _{a_{1} \in A_{n},} a_{2} \in A_{n}, a_{1 \neq a_{2}}\left(\inf _{x \in a_{1}, v \in a_{2}}|x-y|\right),
$$

for $n=1,2,3, \cdots$. Then the following statements are easily checked:

(1) $\sigma A_{n} \subset B$,

(2) $\left(a_{1} \in A_{n}, a_{2} \in A_{n}, a_{1} \neq a_{2}\right) \rightarrow\left(a_{1} \cap a_{2}=\varnothing\right)$ for $n=1,2,3, \cdots$,

(3) $\lim N(n) /\left(\pi n^{2}\right)=1$,

(4) $\quad D_{n}=a / n-a /(n \cdot N(n))-2 a / N(n)$,

(5) $\lim _{n \rightarrow \infty} D_{n} /(a / n)=1$,

(6) $\quad \lim _{n \rightarrow \infty} D_{n}^{2} \cdot N(n) /\left(\pi a^{2}\right)=1$.

6.10 Fundamental Construiction. Let $n_{1}=100$ and define the increasing sequence $n_{1}, n_{2}, \cdots$ inductively so that

$$
N\left(n_{i+1}\right) \geqq M_{i}^{4}, \quad \text { for } i=1,2,3, \cdots,
$$

where for brevity we shall let

$$
M_{i}=\prod_{i=1}^{i} N\left(n_{j}\right), \quad \text { for } i=1,2,3, \cdots
$$

Let

$$
A_{0}=\left\{E^{2} \cap \underset{x}{E}[|x| \leqq 1 / 2]\right\},
$$

and define $A_{i}$ inductively as follows:

Denoting the $n_{i}$-uniform spread of $a \in A_{i-1}$ by $u(a)$, we define

$$
A_{i}=\bigcup_{a \in A_{i-1}} u(a) \quad \text { for } i=1,2,3, \cdots \text {. }
$$

It is obvious that if $a \in A_{i}$, then $a$ is a closed disc in $E^{2}$ of diameter $M_{i}^{-1}$ and that $\gamma\left(A_{i}\right)=M_{i}$ for $i=1,2,3, \cdots$.

For the remainder of this paper we shall let

$$
d_{i}=\inf _{a_{1} \in A_{i}, a_{2} \in A_{i}, a_{1 \neq a_{2}}} \operatorname{dist}\left(a_{1}, a_{2}\right) \quad \text { for } i=1,2,3, \ldots \text {. }
$$

Note that if in 6.9 we let $B$ be some element of $A_{i-1}$, then

$$
D_{n_{i}}=d_{i}
$$

We now define 


$$
A=\bigcap_{i=0}^{\infty} \sigma A_{i}
$$

Since $\sum_{a \in A_{i}} \operatorname{diam} a=1$ for $i=1,2,3, \cdots$, it follows from the definition of $\mathcal{F C}_{2}^{1}$ that $\mathcal{H C}_{2}^{1}(A) \leqq 1$.

We define $B_{i}$ for $i=1,2,3, \cdots$, as follows:

$$
B_{i}=\underset{a \in A_{i}}{\bigcup}\{a \times \underset{y}{E}[0 \leqq y \leqq 1]\} \text {. }
$$

Further let

$$
B=\bigcap_{i=0}^{\infty} \sigma B_{i}
$$

Then

$$
B=A \times \underset{t}{E}[0 \leqq t \leqq 1]
$$

$A$ and $B$ will be fixed for the remainder of this section. Theorem 6.18 will show that $\mathcal{F}_{3}^{2}(B)>1 \geqq \mathcal{F}_{2}^{1}(A)$. But first we need some preliminary definitions and theorems.

6.11 ThEOREM. If $c$ is a complete set, then

$$
0 \leqq \lim _{i \rightarrow \infty} \mathcal{L}_{3}\left(c \cap \sigma B_{i}\right) / \mathcal{L}_{3}\left(\sigma B_{i}\right) \leqq 1
$$

Proof. For $i=1,2,3, \cdots$, define

$$
\psi_{i}(S)=\mathcal{L}_{2}\left(S \cap \sigma A_{i}\right) / \mathcal{L}_{2}\left(\sigma A_{i}\right) \quad \text { for } S \subset E^{2} .
$$

Then $\psi_{i}$ is a measure over $E^{2}, \psi_{i}\left(E^{2}\right)=1$, and hence

$$
\int f d \psi_{i} \leqq\|f\|=\sup _{x \in E^{2}}|f(x)|
$$

for any Baire function $f$ on $E^{2}$ to $E^{1}$.

Let $C_{i}$, for $i=1,2,3, \cdots$, be the set of all functions $f$ on $E^{2}$ to $E^{1}$ such that $(f \mid a)$ is constant for all $a \in A_{i}$. The proof is divided into 4 parts.

Part 1. $\left(f \in C_{i}\right) \rightarrow\left(\lim _{k \rightarrow \infty} \int f d \psi_{k}\right.$ exists $)$.

Proof. Just notice that if $f \in C_{i}$, then $\int f d \psi_{j}=\int f d \psi_{i}$ for $j \geqq i$.

Part 2. If $g$ is such a continuous function on $E^{2}$ that for some $i \geqq 1$ and for all $a \in A_{i}$,

$$
(x \in a, y \in a) \rightarrow(|g(x)-g(y)| \leqq \epsilon),
$$

then for $j \geqq i, k \geqq i$,

$$
\left|\int g d \psi_{j}-\int g d \psi_{k}\right| \leqq \epsilon
$$


Proof. Define $f_{1}, f_{2} \in C_{i}$ as follows:

$$
\begin{array}{ll}
f_{1}(x)=\inf _{\nu \in a} g(y) & \text { if } x \in a \in A_{i}, \\
f_{1}(x)=g(x) & \text { if } x \in E^{2}-\sigma A_{i}, \\
f_{2}(x)=\sup _{\nu \in a} g(y) & \text { if } x \in a \in A_{i}, \\
f_{2}(x)=g(x) & \text { if } x \in E^{2}-\sigma A_{i .} .
\end{array}
$$

Then $f_{1}(x) \leqq g(x) \leqq f_{2}(x)$ for $x \in E^{2},\left\|f_{2}-f_{1}\right\| \leqq \epsilon$. Hence for $k \geqq i$,

$$
\int f_{1} d \psi_{i}=\int f_{1} d \psi_{k} \leqq \int g d \psi_{k} \leqq \int f_{2} d \psi_{k}=\int f_{2} d \psi_{i}
$$

Since

$$
\int f_{2} d \psi_{i}-\int f_{1} d \psi_{i} \leqq\left\|f_{2}-f_{1}\right\| \leqq \epsilon,
$$

we conclude that if $j \geqq i, k \geqq i$, then

$$
\left|\int g d \psi_{j}-\int g d \psi_{k}\right| \leqq \epsilon .
$$

This completes the proof of Part 2.

Part 3. If $g$ is a continuous function on $E^{2}$, then

$$
-\infty<\lim _{k \rightarrow \infty} \int g d \psi_{k}<\infty .
$$

Proof. Note that $g$ is uniformly continuous on the closed unit disc centered at the origin. Hence for any $\epsilon>0$, we can choose $i \geqq 1$ so that for all $a \in A_{i}$,

$$
(x \in a, y \in a) \rightarrow(|g(x)-g(y)| \leqq \epsilon) .
$$

Apply Part 2 to complete the proof.

Part 4. $0 \leqq \lim _{i \rightarrow \infty} \mathcal{L}_{3}\left(c \cap \sigma B_{i}\right) / \mathcal{L}_{3}\left(\sigma B_{i}\right) \leqq 1$.

Proof. Applying 6.6 and Part 3, we have

$$
-\infty<\lim _{i \rightarrow \infty} \int g_{c} d \psi_{i}<\infty .
$$

Since $0 \leqq \int g_{c} d \psi_{i}=\mathcal{L}_{3}\left(c \cap \sigma B_{i}\right) / \mathcal{L}_{3}\left(\sigma B_{i}\right) \leqq 1$ for $i=1,2, \cdots$, the proof is complete.

6.12 Corollary. If $c$ is such a complete set that for some $i \geqq 1$,

$$
\left(a \in A_{i}, x \in a, y \in a\right) \rightarrow\left(\left|g_{c}(x)-g_{c}(y)\right| \leqq \epsilon\right),
$$

then 


$$
\left|\frac{\mathcal{L}_{3}\left(c \cap \sigma B_{i}\right)}{\mathcal{L}_{3}\left(\sigma B_{i}\right)}-\lim _{j \rightarrow \infty} \frac{\mathcal{L}_{3}\left(c \cap \sigma B_{j}\right)}{\mathcal{L}_{3}\left(\sigma B_{j}\right)}\right| \leqq \epsilon .
$$

6.13 Definition. In view of 6.11, we define for $c$ a complete set,

$$
f(c)=\lim _{i \rightarrow \infty} \mathcal{L}_{3}\left(c \cap \sigma B_{i}\right) / \mathcal{L}_{3}\left(\sigma B_{i}\right)
$$

6.14 Convention. We shall let $C$ be the set of all complete sets $c$ such that $f(c) \geqq \frac{1}{2} \cdot \sup _{x \in X} f(x)$, where $X=\underset{x}{E}[x$ is complete and diam $x \leqq \operatorname{diam} c]$.

6.15 Lemma. Let $\lambda>0$. Then there exists an integer $K$ such that if $k \geqq K$, $c \in C$, and

$$
100 M_{k}^{-1} \geqq \operatorname{diam} c \geqq d_{k+1}^{2} \cdot M_{k+1} /(100 \pi),
$$

then

$$
(1-\lambda) \cdot f(c) \leqq \mathcal{L}_{3}\left(c \cap \sigma B_{k+1}\right) / \mathcal{L}_{3}\left(\sigma B_{k+1}\right) \leqq(1+\lambda) \cdot f(c) .
$$

Proof. By 6.9(6) choose $K$ so large that for all $k \geqq K$,

$$
(399 / 400) \cdot d_{k+1}^{2} \leqq \pi \cdot\left(2 M_{k}\right)^{-2}\left(N\left(n_{k+1}\right)\right)^{-1} \leqq(401 / 400) \cdot d_{k+1}^{2},
$$

and

$$
M_{k} \geqq 10^{13} / \lambda
$$

Assume $k \geqq K$ Then

$$
\left(401 M_{k}\right)^{-1} \leqq(100 \pi)^{-1} \cdot d_{k+1}^{2} \cdot M_{k+1} \leqq\left(399 M_{k}\right)^{-1} .
$$

Choose $b \in B_{k}$ with base $a \in A_{k}$ and let $E$ be a right circular cylinder whose axis coincides with the axis of $b$, whose height is $(200 \pi)^{-1} \cdot d_{k+1}^{2} \cdot M_{k+1}$, the radius of whose base is $(400 \pi)^{-1} d_{k+1}^{2} \cdot M_{k+1}$, and which is completely contained in $b$. Then

$$
\operatorname{diam} E \leqq(100 \pi)^{-1} d_{k+1}^{2} \cdot M_{k+1},
$$

and since there are more than $\pi\left((800 \pi)^{-1} \cdot d_{k+1} \cdot M_{k+1}\right)^{2}$ elements of $A_{k+1}$ contained in the closed disc of radius $\left((400 \pi)^{-1} d_{k+1} \cdot M_{k+1}\right) d_{k+1}$ and center coinciding with the center of $a$,

$$
\begin{aligned}
f(E) & \geqq \frac{\left(\pi\left((800 \pi)^{-1} d_{k+1} M_{k+1}\right)^{2}\right):\left(\pi\left(2 M_{k+1}\right)^{-2}\right) \cdot\left((200 \pi)^{-1} d_{k+1}^{2} M_{k+1}\right)}{\left(M_{k+1}\right)\left(\pi\left(2 M_{k+1}\right)^{-2}\right)} \\
& =\left(128 \cdot 100^{3} \cdot \pi^{2}\right)^{-1} \cdot d_{k+1}^{4} \cdot M_{k+1}^{2} .
\end{aligned}
$$


Let now $c \in C$ be such that

$$
100 M_{k}^{-1} \geqq \operatorname{diam} c \geqq(100 \pi)^{-1} \cdot d_{k+1}^{2} \cdot M_{k+1} .
$$

Then

$$
f(c) \geqq\left(256 \cdot 100^{3} \cdot \pi^{2}\right)^{-1} d_{k+1}^{4} \cdot M_{k+1}^{2} .
$$

Also, if $a \in A_{k+1}, x \in a, y \in a$, then by 6.6 ,

$$
\left|g_{c}(x)-g_{c}(y)\right| \leqq 2^{3 / 2} \cdot(\operatorname{diam} c)^{1 / 2}\left(M_{k+1}\right)^{-1 / 2} \leqq 2^{3 / 2} \cdot 10 \cdot M_{k}^{-1} \cdot N\left(n_{k+1}\right)^{-1 / 2} .
$$

Hence by 6.12 ,

$$
\begin{aligned}
\mid\left(\mathcal{L}_{3}\left(c \cap \sigma B_{k+1}\right)\right. & \left./ \mathcal{L}_{3}\left(\sigma B_{k+1}\right)\right)-f(c) \mid \leqq 2^{3 / 2} \cdot 10 \cdot M_{k}^{-1} \cdot N\left(n_{k+1}\right)^{-1 / 2} \\
& \leqq \frac{2^{3 / 2} \cdot 10 \cdot M_{k}^{-1} \cdot N\left(n_{k+1}\right)^{-1 / 2}}{\left(256 \cdot 100^{3} \cdot \pi^{2}\right)^{-1} \cdot d_{k+1}^{4} \cdot M_{k+1}^{2}} \cdot f(c) \\
& =2^{3 / 2} \cdot 256 \cdot 10^{7} \cdot \pi^{2} \cdot f(c) \cdot\left(d_{k+1}^{8} \cdot M_{k}^{6} \cdot N\left(n_{k+1}\right)^{5}\right)^{-1 / 2} \\
& \leqq 10^{11} \cdot \pi^{2} \cdot f(c) \cdot\left(\left(\frac{401}{100 \pi}\right)^{4} \cdot \frac{M_{k}^{8} \cdot N\left(n_{k+1}\right)^{4}}{M_{k}^{6} \cdot N\left(n_{k+1}\right)^{5}}\right)^{1 / 2} \\
& \leqq 10^{13} \cdot f(c) \cdot M_{k} \cdot N\left(n_{k+1}\right)^{-1 / 2} \\
& \leqq 10^{13} \cdot f(c) \cdot M_{k}^{-1} \\
& \leqq \lambda \cdot f(c) .
\end{aligned}
$$

The proof is complete.

6.16 Lemma. Let $\lambda>0$. There exists an integer $K$ such that if $k \geqq K$, then

(1) $100 \cdot M_{\mathrm{k}}^{-1} \leqq d_{k}$,

(2) $\left(401 \cdot M_{k}\right)^{-1} \leqq(100 \pi)^{-1} d_{k+1}^{2} \cdot M_{k+1} \leqq\left(399 \cdot M_{k}\right)^{-1}$,

(3) If $c$ is a complete set which is also complete with respect to some $b \in B_{k}$, $\operatorname{diam} c=\operatorname{diam}(c \cap b)$, and

$$
100 M_{k}^{-1} \geqq \operatorname{diam} c \geqq(100 \pi)^{-1} \cdot d_{k+1}^{2} \cdot M_{k+1},
$$

then

$$
(1-\lambda) \leqq\left(\frac{\mathcal{L}_{3}\left(c \cap \sigma B_{k+1}\right)}{\mathcal{L}_{3}\left(\sigma B_{k+1}\right)}\right) \cdot\left(\frac{\mathcal{L}_{3}\left(c \cap \sigma B_{k}\right)}{\mathcal{L}_{3}\left(\sigma B_{k}\right)}\right)^{-1} \leqq(1+\lambda) .
$$

Proof. Let $q$ be the infimum of the volumes of all complete sets of diameter 1. Then $q>0$. By $6.9(6)$, (5), (3), choose $K$ so large that for all $k \geqq K$, (1) and (2) of this lemma hold, and $M_{k} \geqq 10^{14} \cdot q^{-1} \cdot \lambda^{-1}$.

Let $k \geqq K$, and suppose $c$ satisfies the hypotheses of (3). Then since diam $c \leqq d_{k}$, we see that $c$ intersects exactly one element of $B_{k}$, namely $b$. 
Because $c$ is complete with respect to $b$, diam $(c \cap b)=\operatorname{diam} c \geqq(100 \pi)^{-1}$ $\cdot d_{k+1}^{2} \cdot M_{k+1}$, we have

$$
\mathcal{L}_{3}\left(c \cap \sigma B_{k}\right) \geqq q\left((100 \pi)^{-1} \cdot d_{k+1}^{2} \cdot M_{k+1}\right)^{3} .
$$

Let $a \in A_{k}$ be the base of $b$, and suppose $\alpha_{1}, \cdots, \alpha_{N\left(n_{k+1}\right)}$ are the $N\left(n_{k+1}\right)$ elements of $A_{k+1}$ contained in $a$. Now associate to each $\alpha_{i}$ for $i=1, \cdots$, $N\left(n_{k+1}\right)$, a Borel set $\alpha_{i}^{\prime} \subset a$ such that

$$
\begin{aligned}
& \alpha_{i}^{\prime} \cap \alpha_{j}^{\prime}=\varnothing \quad \text { for } \quad i \neq j, \quad \alpha_{i} \subset \alpha_{i}^{\prime}, \quad \operatorname{diam} \alpha_{i}^{\prime} \leqq 2 d_{k+1}, \\
& \bigcup_{i=1}^{N\left(n_{k+1}\right)} \alpha_{i}^{\prime}=a, \quad \mathcal{L}_{2}\left(\alpha_{i}^{\prime}\right)=\pi \cdot\left(2 M_{k}\right)^{-2} \cdot N\left(n_{k+1}\right)^{-1} .
\end{aligned}
$$

If $x \in \alpha_{i}^{\prime}, y \in \alpha_{i}^{\prime}$ for some $1 \leqq i \leqq N\left(n_{k+1}\right)$, then by 6.6 ,

$$
\left|g_{c}(x)-g_{c}(y)\right| \leqq 2^{3 / 2} \cdot\left(2 \cdot d_{k+1} \cdot \operatorname{diam} c\right)^{1 / 2} \leqq 40 \cdot\left(d_{k+1} \cdot M_{k}^{-1}\right)^{1 / 2},
$$

and hence

$$
\begin{aligned}
& \left|\int_{\alpha_{i}^{\prime}} g_{c}(x) d \mathcal{L}_{2} x-N\left(n_{k+1}\right) \cdot \int_{\alpha_{i}} g_{c}(x) d \mathcal{L}_{2} x\right| \leqq 40 \cdot d_{k+1}^{1 / 2} \cdot M_{k}^{-1 / 2} \cdot \mathcal{L}_{2}\left(\alpha_{i}^{\prime}\right), \\
& \quad\left|\mathcal{L}_{3}\left(c \cap \sigma B_{k}\right)-N\left(n_{k+1}\right) \mathcal{L}_{3}\left(c \cap \sigma B_{k+1}\right)\right| \leqq 40 \cdot d_{k+1}^{1 / 2} \cdot M_{k}^{-1 / 2} \cdot \pi \cdot\left(2 M_{k}\right)^{-2} .
\end{aligned}
$$

Therefore,

$$
\begin{aligned}
&\left|1-\left(\frac{\mathcal{L}_{3}\left(c \cap \sigma B_{k+1}\right)}{\mathcal{L}_{3}\left(\sigma B_{k+1}\right)}\right) \cdot\left(\frac{\mathcal{L}_{3}\left(c \cap \sigma B_{k}\right)}{\mathcal{L}_{3}\left(\sigma B_{k}\right)}\right)^{-1}\right| \\
&=\left|1-\frac{\mathcal{L}_{3}\left(c \cap \sigma B_{k+1}\right) \cdot N\left(n_{k+1}\right)}{\mathcal{L}_{3}\left(c \cap \sigma B_{k}\right)}\right| \\
& \leqq \frac{40 \cdot d_{k+1}^{1 / 2} \cdot M_{k}^{-1 / 2} \cdot \pi \cdot\left(2 M_{k}\right)^{-2}}{q \cdot\left[(100 \pi)^{-1} \cdot d_{k+1}^{2} \cdot M_{k+1}\right]^{3}} \\
&=\frac{(100 \pi)^{3}}{q} \cdot 10 \pi \cdot\left(d_{k+1}^{11} \cdot M_{k}^{11} \cdot N\left(n_{k+1}\right)^{6}\right)^{-1 / 2} \\
& \leqq \frac{10^{11}}{q}\left(\left(\frac{401}{100 \pi}\right)^{11 / 2} \cdot \frac{M_{k}^{11} \cdot N\left(n_{k+1}\right)^{11 / 2}}{M_{k}^{11} \cdot N\left(n_{k+1}\right)^{6}}\right)^{1 / 2} \\
& \leqq \frac{10^{14}}{q} \cdot M_{k}^{-1} \leqq \lambda .
\end{aligned}
$$

The proof is complete.

6.17 Lemma. Let $\lambda>1$. There exists an integer $K$ such that if $k \geqq K$, then 
(1) $d_{k} \geqq 100 M_{k}^{-1}$,

(2) $\left(401 \cdot M_{k}\right)^{-1} \leqq(100 \pi)^{-1} \cdot d_{k+1}^{2} \cdot M_{k+1} \leqq\left(399 \cdot M_{k}\right)^{-1}$, and

(3) If $c \in C$ is complete with respect to some $b \in B_{k}$, diam $(b \cap c)=\operatorname{diam} c$,

$$
100 M_{k}^{-1} \geqq \operatorname{diam} c \geqq(100 \pi)^{-1} d_{k+1}^{2} \cdot M_{k+1},
$$

then

$$
\lambda^{-1} \cdot f(c) \leqq \mathcal{L}_{3}\left(c \cap \sigma B_{k}\right) / \mathcal{L}_{3}\left(\sigma B_{k}\right) \leqq \lambda \cdot f(c) .
$$

Proof. Apply 6.15, 6.16.

6.18 ThEOREM. $\mathfrak{F C}_{3}^{2}(B)>1 \geqq \mathfrak{F C}_{2}^{1}(A)$.

Proof. Note that in the definition of $\mathcal{F}_{3}^{2}$, we may restrict our coverings to consist of complete sets. We can do this because every set in $E^{3}$ is contained in a complete set of the same diameter.

Let $\lambda>1$. Choose $K$ as in 6.17. Then the theorem follows from Part 4 and the arbitrary nature of $\lambda$.

Part 1 . If $k \geqq K, c$ is such a complete set that

$$
100 M_{k}^{-1} \leqq \operatorname{diam} c \leqq d_{k}, \quad f(c)>0,
$$

then

$$
(\operatorname{diam} c)^{2} / f(c) \geqq 100 .
$$

Proof. Since diam $c \leqq d_{k}$ and $f(c)>0, c$ intersects exactly one element of $B_{k}$, say $b$. Let

$$
\tau=\inf \underset{t}{E}\left[\left(x_{1}, x_{2}, t\right) \in(b \cap c) \text { for some } x_{1}, x_{2}\right] .
$$

Then

$$
(b \cap c) \subset\left(b \cap \underset{x}{E}\left[\tau \leqq x_{3} \leqq \tau+\operatorname{diam} c\right]\right),
$$

and hence

$$
\begin{aligned}
f(c) & \leqq \operatorname{diam} c \cdot M_{k}^{-1}, \\
(\operatorname{diam} c)^{2} / f(c) & \geqq M_{k} \cdot \operatorname{diam} c \geqq 100 .
\end{aligned}
$$

This completes the proof of Part 1 .

Part 2. If $k \geqq K, c$ is such a complete set that

$$
d_{k+1} \leqq \operatorname{diam} c \leqq(100 \pi)^{-1} \cdot d_{k_{k+1}}^{2} \cdot M_{k+1}, \quad f(c)>0,
$$

then

$$
(\operatorname{diam} c)^{2} / f(c) \geqq 25 \text {. }
$$


Proof. The set $c$ cannot intersect more than $\pi\left(2 \cdot \operatorname{diam} c / d_{k+1}\right)^{2}$ elements of $B_{k+1}$, since $p_{3}^{2 *}(c)$ is contained in a disc of radius equal to diam $c$. Hence, by a similar argument as was used in the proof of Part 1, namely that the intersection of $c$ with an element of $B_{k+1}$ is contained in a cylinder of height equal to diam $c$ and base radius equal to $\left(2 M_{k+1}\right)^{-1}$, we conclude that

$$
f(c) \leqq \pi\left(2 \cdot \operatorname{diam} c / d_{k+1}\right)^{2} \cdot \operatorname{diam} c \cdot M_{k+1}^{-1}
$$

and therefore

$$
(\operatorname{diam} c)^{2} / f(c) \geqq d_{k+1}^{2} \cdot M_{k+1} /(4 \pi \cdot \operatorname{diam} c) \geqq 25 .
$$

This proves Part 2.

Part 3. If $k \geqq K, c$ is such a complete set that

$$
(100 \pi)^{-1} \cdot d_{k+1}^{2} \cdot M_{k+1} \leqq \operatorname{diam} c \leqq 100 M_{k}^{-1}, \quad f(c)>0,
$$

then

$$
(\operatorname{diam} c)^{2} / f(c) \geqq 2 \cdot\left(108^{1 / 4}-12^{1 / 4}\right)^{-1} \cdot \lambda^{-1} .
$$

Proof. If $c \notin C$, then we can choose a set $c^{\prime} \in C$ such that $\operatorname{diam} c=\operatorname{diam} c^{\prime}$, and $f\left(c^{\prime}\right)>f(c)$. Hence

$$
(\operatorname{diam} c)^{2} / f(c)>\left(\operatorname{diam} c^{\prime}\right)^{2} / f\left(c^{\prime}\right),
$$

so that we may just as well assume that $c \in C$.

Since diam $c \leqq d_{k}, c$ intersects exactly one element of $B_{k}$, say $b$. Now there exists a complete set $c^{\prime}$ such that

$(c \cap b) \subset\left(c^{\prime} \cap b\right), \quad \operatorname{diam}\left(c^{\prime} \cap b\right)=\operatorname{diam} c=\operatorname{diam} c^{\prime}$, $c^{\prime}$ is complete with respect to $b$.

Hence $f\left(c^{\prime}\right) \geqq f(c),(\operatorname{diam} c)^{2} / f(c) \geqq\left(\operatorname{diam} c^{\prime}\right)^{2} / f\left(c^{\prime}\right)$, and so we may assume that $c \in C$ is complete with respect to $b$ and that diam $(b \cap c)=\operatorname{diam} c$.

Applying 6.17, we have

$$
\lambda^{-1} \cdot f(c) \leqq \mathcal{L}_{3}\left(c \cap \sigma B_{k}\right) / \mathcal{L}_{3}\left(\sigma B_{k}\right) \leqq \lambda \cdot f(c),
$$

and therefore

$$
\lambda^{-1} \cdot(\operatorname{diam} c)^{2} / f(c) \leqq(\operatorname{diam} c)^{2} \cdot \mathcal{L}_{3}\left(\sigma B_{k}\right) / \mathcal{L}_{3}\left(c \cap \sigma B_{k}\right) \leqq \lambda \cdot(\operatorname{diam} c)^{2} / f(c) .
$$

By 6.3, if $s$ is a sphere of diameter equal to diam $c$, with center on the axis of $b$, and which does not meet either base of $b$, then

$$
(\operatorname{diam} c)^{2} \cdot \mathcal{L}_{3}\left(\sigma B_{k}\right) / \mathcal{L}_{3}\left(c \cap \sigma B_{k}\right) \geqq(\operatorname{diam} s)^{2} \cdot \mathcal{L}_{3}\left(\sigma B_{k}\right) / \mathcal{L}_{3}\left(s \cap \sigma B_{k}\right) .
$$

Our problem is thus reduced to the following calculus problem:

Let 


$$
E=E^{3} \cap \underset{x}{E}\left[x_{1}^{2}+x_{2}^{2} \leqq r^{2}\right]
$$

then find the number $s$ such that

$$
r / 401 \leqq s \leqq 100 r \text {, and } 4 s^{2} / \mathcal{L}_{3}(E \cap \underset{x}{E}[|x| \leqq s]) \text { is least. }
$$

It is easily checked that $s=r \cdot 3^{-1 / 4} \cdot 2^{1 / 2}$, and that

$$
4 s^{2} / \mathcal{L}_{3}(E \cap \underset{x}{E}[|x| \leqq s])=4\left(\pi r\left(108^{1 / 4}-12^{1 / 4}\right)\right)^{-1} .
$$

Hence

$$
\begin{aligned}
(\operatorname{diam} c)^{2} / f(c) & \geqq \lambda^{-1} \cdot(\operatorname{diam} s)^{2} \cdot \mathcal{L}_{3}\left(\sigma B_{k}\right) / \mathcal{L}_{3}\left(s \cap \sigma B_{k}\right) \\
& \geqq \lambda^{-1} \cdot 4\left(\pi\left(108^{1 / 4}-12^{1 / 4}\right) / 2 \cdot M_{k}\right)^{-1} \cdot\left(\pi M_{k} 4 M_{k}^{2}\right) \\
& =2 \cdot\left(108^{1 / 4}-12^{1 / 4}\right)^{-1} \cdot \lambda^{-1} .
\end{aligned}
$$

This proves Part 3 .

Part 4. $\mathcal{F}_{3}^{2}(B) \geqq \lambda^{-1} \cdot \pi \cdot\left(2\left(108^{1 / 4}-12^{1 / 4}\right)\right)^{-1}>(1.1) \cdot \lambda^{-1}$.

Proof. Recall that $B$ is compact and hence we may assume that any covering of $B$ by complete sets of positive diameter is finite. Let $F$ be such a finite covering of $B$ by complete sets whose diameters are less than $d_{K}$. Then by Parts 1, 2, and 3,

$$
(c \in F \text { and } f(c) \neq 0) \rightarrow\left((\operatorname{diam} c)^{2} / f(c) \geqq 2\left(108^{1 / 4}-12^{1 / 4}\right)^{-1} \cdot \lambda^{-1}\right) .
$$

Also by the definition of $f$, it is obvious that

$$
\sum_{c \in F} f(c) \geqq 1 \text {. }
$$

Hence

$$
\begin{aligned}
\sum_{c \in F}(\pi / 4)(\operatorname{diam} c)^{2} & \geqq 2\left(108^{1 / 4}-12^{1 / 4}\right)^{-1} \cdot \lambda^{-1} \cdot(\pi / 4) \sum_{c \in F} f(c) \\
& \geqq \pi \cdot 2^{-1} \cdot\left(108^{1 / 4}-12^{1 / 4}\right)^{-1} \cdot \lambda^{-1} \\
& >(1.1) \cdot \lambda^{-1} .
\end{aligned}
$$

By the definition of $\mathfrak{H}_{3}^{2}$, we conclude that

$$
\mathfrak{F C}_{3}^{2}(B) \geqq \pi \cdot 2^{-1} \cdot\left(108^{1 / 4}-12^{1 / 4}\right)^{-1} \cdot \lambda^{-1}>(1.1) \cdot \lambda^{-1} \text {. }
$$

The proof is complete.

6.19 Corollary. $S_{3}^{2}(B)>S_{2}^{1}(A)$.

Proof. It is easy to see that in general,

$$
\mathcal{S}_{n}^{k}(X) \geqq \mathcal{F C}_{n}^{k}(X) \quad \text { for } X \subset E^{n}, 0 \leqq k \leqq n .
$$


Moreover it is obvious that $\mathcal{S}_{2}^{1}(A) \leqq 1$, hence

$$
\mathcal{S}_{3}^{2}(B) \geqq \mathcal{F C}_{3}^{2}(B)>1 \geqq \mathcal{S}_{2}^{1}(A),
$$

so that for sphere measure, it is likewise not true that the measure of a cylinder set is the product of the measure of the base by the height.

6.20 REMARK. Since $\mathcal{F}_{3}^{2}(B)>0$, it follows that $\mathcal{F}_{2}^{1}(A)>0$.

6.21 REMARK. By $[R, 5]$, we have

$$
C_{3}^{2}(B) \leqq C_{2}^{1}(A)
$$

Since $C_{2}^{1}(A) \leqq 1$, we conclude that

$$
C_{3}^{2}(B) \leqq 1<\mathfrak{H}_{3}^{2}(B),
$$

thus proving that $C_{3}^{2} \neq \mathcal{F C}_{3}^{2}$.

6.22 REMARK. By means of the construction in 6.10 , examples can immediately be constructed in $E^{n}$ for $n>3$, for which the Hausdorff measure of a product set is not equal to the product of the measures of the components.

\section{BIBLIOGRAPHY}

A. S. Besicovitch and P. A. P. Moran

[BM] The measure of product and cylinder sets, J. London Math. Soc. vol. 20 (1945) pp. 110-120.

J. BONNESEN and W. FENCHEL

[BF] Theorie der konvexen Körper, Ergebnisse der Mathematik, vol. 3, part 1, 1934.

C. Caratheodory

[C] Über das lineare Mass von Punktmengen-eine Verallgemeinerung des Längenbegriffs, Nachr. Ges. Wiss. Göttingen (1914) pp. 404-426.

\section{H. FEDERER}

[F1] Coincidence functions and their integrals, Trans. Amer. Math. Soc. vol. 59 (1946) pp. 441-466.

[F2] The $(\phi, k)$ rectifiable subsets of $n$ space, Trans. Amer. Math. Soc. vol. 62 (1947) pp. 114-192.

[F3] Dimension and measure, Trans. Amer. Math. Soc. vol. 62 (1947) pp. 536-547.

F. HAUSDORFF

[H] Dimension und aiisseres Mass, Math. Ann. vol. 79 (1918) pp. 157-179.

L. H. LOOMIS

[L] Abstract congruence and the uniqueness of Haar measure, Ann. of Math. vol. 46 (1945) pp. 348-355.

A. P. MORSE and H. FEDERER

[MF] Some properties of measurable functions, Bull. Amer. Math. Soc. vol. 49 (1943) pp. 270-277.

A. P. Morse and J. F. RaNdolPH

[MR] Gillespie measure, Duke Math. J. vol. 6 (1940) pp. 408-419.

H. RADEMACHER

[RAD] Über partielle und totale Differenzierbarkeit. I, Math. Ann. vol. 79 (1919) pp. 340-359. 
J. F. RANDOLPH

[R] On generalizations of length and area, Bull. Amer. Math. Soc. vol. 42 (1936) pp. 268-274.

S. SAKS

[S] Theory of the integral, Warsaw, 1937.

A. WeIL

[W] L'intégration dans les groupes topologiques et ses applications, Actualités Scientifiques et Industrielles, vol. 869, Hermann, Paris, 1938.

\section{BROWN UNIVERSITY,}

Providence, R. I. 